\title{
Mandenkan
}

MANDENIKAN Bulletin semestriel d'études linguistiques mandé

$61 \mid 2019$

Numéro 61

\section{Tonologie du mandinka du Pakaawu}

Tonology of the Pakaawu Mandinka

ТОНОЛОГИЯ МАНДИНКА Р-НА ПАКАУ

\section{Denis Creissels}

\section{(2) OpenEdition}

Journals

Édition électronique

URL : https://journals.openedition.org/mandenkan/1886

DOI : 10.4000/mandenkan. 1886

ISSN : 2104-371X

Éditeur

Llacan UMR 8135 CNRS/Inalco

Référence électronique

Denis Creissels, « Tonologie du mandinka du Pakaawu », Mandenkan [En ligne], 61 | 2019, mis en ligne le 30 janvier 2020, consulté le 08 juillet 2021. URL : http://journals.openedition.org/mandenkan/1886 ; DOI : https://doi.org/10.4000/mandenkan.1886

\section{cc) (†) (ㄱ)}

Les contenus de Mandenkan sont mis à disposition selon les termes de la Licence Creative Commons Attribution - Pas d'Utilisation Commerciale - Partage dans les Mêmes Conditions 4.0 International. 


\title{
Tonologie du mandinka du Pakaawu
}

\author{
Denis Creissels \\ (Université de Lyon) \\ denis.creissels@univ-lyon2.fr,http://deniscreissels.fr
}

avec la collaboration de

Yaya Dramé

\section{Introduction}

Cet article a pour objectif une description détaillée de la tonologie du mandinka du Pakaawu. Il met l'accent sur un exposé aussi précis et exhaustif que possible des données pertinentes pour l'analyse du système tonal de ce parler, avec une attention particulière aux processus tonals qui font sa spécificité. Son intérêt essentiel est d'offrir une description détaillée de phénomènes tonals qui jusqu'ici n'ont jamais été mentionnés dans la littérature sur les systèmes tonals mandingues, ou qui ont été seulement entrevus dans le chapitre de tonologie de la description que donnent Creissels et Sambou (2013) du mandinka de Sédhiou. Nous reviendrons d'ailleurs en conclusion sur la signification qu'il convient d'accorder aux divergences entre cette description et le système décrit ici.

Le Pakaawu ou Pakao (Pàkáawù) n'est pas une circonscription administrative, mais un groupement de villages partageant une histoire et une tradition commune qui les distinguent des zones mandingophones voisines. Ce groupe de villages est situé en Moyenne Casamance, à une quarantaine de kilomètres au nord-est de la capitale régionale Sédhiou. Les trois principaux villages du Pakaawu sont Dassilamé, Soumboundou et Mankonomba, désignés traditionnellement comme Pàkáawú sìnkírí sàbôo 'les trois pierres du foyer du Pakaawu'. La spécificité historique de cette zone est d'avoir été très tôt un foyer d'islamisation et de mandinguisation, et d'avoir joué un rôle important dans les conflits avec les Baïnounk animistes (Roche 1985). Une des particularités du Pakaawu est de n'avoir pas connu le système de la royauté (mànsàyâa), les villages étant dirigés traditionnellement par des notables désignés du nom de kàndàalú. Les habitants du Pakaawu se targuent d'être des connaisseurs de l'islam, et leurs marabouts sont célèbres pour leur maîtrise des pratiques magiques qui 
lui sont liées. Le lecteur intéressé par ces questions consultera avec profit (Schaffer 2003).

Le travail présenté ici est le résultat d'un travail régulier, sur une durée de six ans, avec Yaya Dramé, originaire du village de Dassilamé, résidant actuellement à Lyon où il enseigne l'anglais. De 2013 à 2016, j'ai supervisé ses travaux dans le cadre du master de Sciences du Langage de l'Université Lyon 2, qui ont abouti à un mémoire sur les particularités phonologiques et morphosyntaxiques des noms propres de personnes en mandinka du Pakaawu (Dramé 2016). Cela a été l'occasion de lui faire acquérir une parfaite maitrise de la transcription du mandinka, y compris sous l'aspect tonal. Seule la conscience qu'il a acquise des moindres détails de ses réalisations tonales a permis à ce travail d'être ce qu'il est, car plus d'une fois, c'est son insistance à rectifier ce que je croyais avoir perçu de prime abord qui m'a conduit à préciser ou même à revoir complètement certaines analyses, notamment en ce qui concerne la présence systématique d'un ton modulé descendant sur la syllabe finale des verbes à syllabe finale lourde. Depuis la soutenance de son mémoire de master, nous avons entrepris en commun la vérification systématique des données lexicales que j'avais recueillies précédemment sur le mandinka, en vue d'un dictionnaire mandinka-français que nous espérons publier en 2020. La vérification tonale systématique, non seulement des entrées lexicales, mais aussi et surtout des phrases illustratives, qui figureront toutes dans le dictionnaire avec une transcription phonétique intégrale de leur contour tonal, a fourni l'essentiel du matériau dont l'analyse est présentée ici.

Le mandinka est dans le domaine lexical et morphosyntaxique, ainsi que dans le domaine de la phonologie segmentale, une langue remarquablement homogène relativement à l'étendue du territoire sur lequel elle est parlée, de l'est de la Guinée Bissau à l'embouchure de la Gambie. Par conséquent, compte tenu de la proximité géographique entre Sédhiou et le Pakaawu, lorsque j'ai commencé à travailler avec Yaya Dramé, je ne m'attendais pas à ce que son parler diffère de manière notable de celui de Sédhiou sur lequel j'avais travaillé précédemment. De manière générale, cette prédiction s'est vérifiée, sauf précisément dans le domaine tonal, et c'est qui a motivé l'écriture de cet article.

L'article est organisé de la façon suivante. La section 2 donne un aperçu des caractéristiques saillantes du système tonal du mandinka du Pakaawu. La section 3 discute la question des « tons hauts faibles ». La section 4 discute l'alternance tonale $\mathrm{H} \mathrm{H} \sim \mathrm{H} \mathrm{B}$ en fin de mot et le relèvement tonal. La section 5 analyse la relation entre ton et poids syllabique, et les contraintes sur les séquences tonales possibles dans les mots de deux syllabes ou plus. La section 6 décrit les particularités tonales de mots monosyllabiques. Les sections 7 et 8 sont consacrées respectivement au ton dans le système nominal et au ton dans le système verbal. La section 9 résume les principales conclusions. 
Les abréviations utilisées dans la description des processus tonals, la schématisation des structures syllabiques et le glosage des exemples sont données à la fin de l'article.

\section{Quelques caractéristiques saillantes du système tonal du mandinka du Pakaawu}

Cet article a comme premier objectif de documenter la variation dialectale dans les systèmes de tons mandingues. Il s'adresse donc en priorité à des lecteurs intéressés par la tonologie mandingue et connaissant plus ou moins les systèmes tonals des variétés mandingues déjà documentées sur le plan tonal. Dans ces conditions, il est utile de commencer par un aperçu général de ce que le système tonal du mandinka du Pakaawu a en commun avec celui des variétés mandingues les mieux connues, et de ce qui fait par contre sa spécificité. Les variétés de référence sont essentiellement le bambara standard (Dumestre 2003; Vydrin 2017), le maninka-mori (Grégoire 1986) et le maninka de Kita (Creissels 2009). Lorsqu'ils méritent un exposé plus détaillé, les phénomènes brièvement évoqués dans la deuxième partie de cette section seront repris en détail dans la suite de l'article.

\subsection{Points communs entre le système tonal du mandinka du Pakaawu et} celui des autres variété $\mathbf{s}$ mandingues dont le système tonal est documenté

\subsubsection{L'opposition $H$ vs. $B$}

Comme de manière générale en mandingue, le mandinka du Pakaawu a un système tonal analysable sans problème comme basé sur une opposition binaire $\mathrm{H}$ vs. B. Une preuve particulièrement claire de cette opposition est le contraste entre $i$ pronom de 2ème personne du singulier et $i$ pronom de troisième personne du pluriel, comme en (1) et (2).

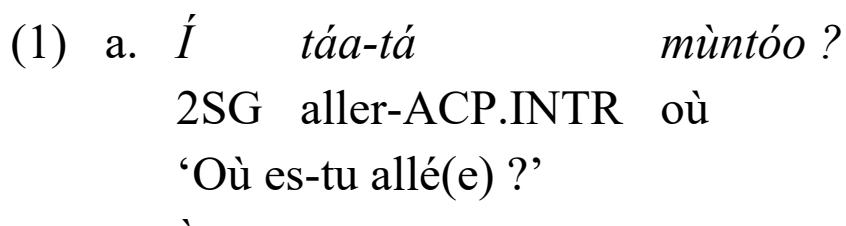
b. İ táa-tá mùntóo?
3PL aller-ACP.INTR où
'Où sont-ils/elles allé(e)s ?'
(2) a. Í năa-tà mú nè lá ?
2SG venir-ACP.INTR quoi FOC POSTP
'Pourquoi es-tu venu(e)?'
b. Ì năa-tà mú nè lá?
3PL venir-ACP.INTR quoi FOC POSTP
'Pourquoi sont-ils/elles venu(e)s?'




\subsubsection{Le downdrift}

Comme c'est la règle générale en mandingue, la réalisation des séquences de tons $\mathrm{H}$ et de tons $\mathrm{B}$ en mandinka du Pakaawu est conditionnée par un phénomène automatique de downdrift : lorsque deux séquences de tons $\mathrm{H}$ appartenant au même groupe prosodique sont séparées l'une de l'autre par un ou plusieurs tons bas, la deuxième est réalisée à un niveau inférieur à la première : ${ }^{1}$

$\ldots \mathrm{H}^{\mathrm{x}} \mathrm{B}^{\mathrm{x}} \mathrm{H}^{\mathrm{x}} \ldots \rightarrow \ldots \mathrm{H}^{\mathrm{x}} \mathrm{B}^{\mathrm{x}} \mathrm{H}^{\mathrm{x}} \ldots$

Comme il est totalement automatique, ce phénomène n'a pas besoin d'être noté. Il est toutefois bon de signaler qu'en mandinka du Pakaawu, le downdrift est particulièrement marqué, avec comme conséquence qu'en fin de groupe prosodique, il est difficile de percevoir la distinction entre tons $\mathrm{H}$ et tons $\mathrm{B}$.

\subsubsection{Stabilité du ton initial et variabilité du ton final des mots}

En mandinka du Pakaawu, comme en bambara standard, en maninka-mori ou en maninka de Kita, le ton final des mots peut varier en fonction du ton initial du mot suivant, alors que le ton initial des mots n'est jamais affecté par le sandhi tonal (à la différence par exemple du bambara de Ségou, ou du parler mandinka de Gambie centrale sur la tonologie duquel Rowlands (1959) fournit des données).

En outre, à très peu d'exceptions près, le ton initial des mots en mandinka du Pakaawu est le même qu'en bambara standard, en maninka-mori ou en maninka de Kita. Par exemple, les deux noms dont la forme de citation en bambara est kúrûn 'bateau' et kòlôn mortier sont cités en mandinka du Pakaawu comme kúlúyò 'bateau' et kùlúyò 'mortier'. ${ }^{2}$ Par contre, ce qui se passe tonalement à la fin des mots en mandinka du Pakaawu est à bien des égards très différent de ce qui s'observe ailleurs en mandingue.

\subsubsection{Compacité tonale}

Comme les autres variétés de mandingue dont le système tonal est documenté, le mandinka du Pakaawu a un phénomène de compacité tonale qui opère lors de la formation des lexèmes complexes, selon lequel la tonalité du lexème complexe est entièrement déterminée par sa division en constituants immédiats et le ton initial de son premier constituant.

Par rapport au bambara standard, au maninka-mori ou au maninka de Kita, la seule différence notable dans les constructions à compacité tonale concerne le ton des syllabes finales légères. En effet, compte tenu du comportement tonal des syllabes en

${ }^{1}$ Le groupe prosodique est défini ici comme un fragment de discours délimité par deux pauses et qu'aucune pause n'interrompt.

${ }^{2}$ băy 'finir (bambara bán) est l'une des très rares exceptions à cette régularité. 
fin de mot et de l'analyse qui en est donnée ici, il convient de formuler comme suit la règle de compacité tonale en mandinka du Pakaawu :

- le premier constituant immédiat du composé prend une tonalité uniformément haute ou uniformément basse selon que son ton initial est $\mathrm{H}$ ou B ;

-si le deuxième constituant immédiat a une syllabe légère pour syllabe finale, son schème tonal est $\mathrm{H}$ jusqu'à la syllabe pénultième, $\mathrm{B}$ pour la syllabe finale; si la syllabe finale du deuxième constituant immédiat est lourde, il prend une tonalité uniformément haute.

Par exemple, tùbáabù 'européen' + kitáabù 'livre' donne tùbàabù-kítáabù, alors que tùbáabù 'européen' + kàràmbún 'école' donne tùbàabù-kárámbún.

Il y a aussi quelques différences mineures qu'on peut expliquer en posant une division des composés complexes en constituants immédiats qui n'est pas toujours identique à celle que l'on a dans d'autres variétés mandingues. Ceci concerne essentiellement les noms composés dont le deuxième constituant est un nom d'agent, comme fòlèe-sùu-dádáa-láa 'réparateur de bicyclettes', qui correspond au bambara nègè-sò-dlàn-ná.

Il ne sera donc pas utile de revenir sur le phénomène de compacité tonale dans la suite de cet article.

\subsection{Spécificités du système tonal du mandinka du Pakaawu}

\subsubsection{L'absence de downstep systématique}

A la différence de beaucoup de variétés mandingues (notamment le bambara standard, le maninka-mori ou le maninka de Kita), le mandinka du Pakaawu n'a pas un phénomène systématique de downstep (abaissement du registre $\mathrm{H}$ imputable à un ton B sous-jacent qui n'est pas réalisé comme tel mais laisse comme trace de sa présence l'abaissement du registre $\mathrm{H}$ ).

En mandinka du Pakaawu, le downstep apparaît seulement de façon accidentelle, comme réalisation possible d'une séquence $\widehat{\mathrm{HB}} \mathrm{H}$ en débit rapide (et seulement en débit rapide). Normalement, une telle séquence est réalisée comme $\widehat{\mathrm{HB}} ! \mathrm{H}$, avec une modulation descendante bien perceptible et un abaissement automatique du ton $\mathrm{H}$ suivant selon le principe du downdrift, comme en (3a), mais en débit rapide, il est possible que la modulation descendante s'atténue au point de devenir imperceptible, l'abaissement tonal étant alors la seule trace de la présence d'un élément $\mathrm{B}$, comme en (3b). En mandinka du Pakaawu, on peut ainsi rendre compte des séquences $\mathrm{H}{ }^{\prime} \mathrm{H}$ par une règle facultative qui peut modifier les séquences $\widehat{\mathrm{HB}} \mathrm{H}$ en fonction de la rapidité du débit :

$\widehat{\mathrm{HB}} \mathrm{H} \rightarrow \mathrm{H}{ }^{\prime} \mathrm{H}$ 
(3)

a. Mùsôo táa-tá mùntóo?

femme.D aller-ACP.INTR où

'Où la femme est-elle allée ?' (débit normal)

b. Mùsóo 'táa-tá mùntóo?

femme.D aller-ACP.INTR où

'Où la femme est-elle allée ?' (débit rapide)

\subsubsection{Ton et structure syllabique}

Le mandinka du Pakaawu ne présente pas les phénomènes qui, dans d'autres parlers mandingues, peuvent suggérer le regroupement de syllabes en 'pieds' dont la reconnaissance est pertinente pour l'analyse des fonctionnements tonals, ${ }^{3}$ par contre le poids syllabique joue un rôle considérable dans le système tonal du mandinka du Pakaawu.

En mandinka du Pakaawu, outre le mot, les unités pertinentes pour la description et l'analyse des fonctionnements tonals sont le groupe prosodique, le mot, la syllabe et la more. Les syllabes lourdes (CVV ou CVN) n'ont pas les mêmes propriétés tonales que les syllabes légères $(\mathrm{CV})$, et le mandinka du Pakaawu se caractérise par une relation extrêmement stricte entre ton et structure syllabique, encore plus stricte par exemple que dans le mandinka de Sédhiou tel que décrit dans Creissels \& Sambou (2013). Cette question sera reprise de façon détaillée à la section 5. Dans une formalisation de l'analyse développée dans cet article, il est évident que c'est la more qu'il conviendrait de définir comme l'unité porteuse de ton.

Dans cet article, les syllabes à ton modulé sont notées comme suit : $\mathrm{CVV}, \mathrm{C} \hat{\mathrm{V} N}$,

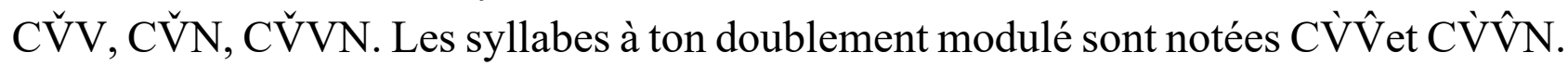

\subsubsection{Alternances tonales conditionnées phonologiquement}

\subsubsection{Séquences tonales B $H$ précédant immédiatement une frontière}

Les variétés mandingues les mieux connues ont une alternance systématique entre séquences tonales entièrement basses et séquences tonales ascendantes, dont l'analyse a d'ailleurs donné lieu à une variété d'interprétations. En mandinka du Pakaawu, les données phonétiques sont radicalement différentes.

En mandinka du Pakaawu, le ton haut final des unités dont la réalisation tonale alterne en bambara par exemple entre $\mathrm{B}^{\mathrm{x}}$ et $\mathrm{B}^{\mathrm{x}} \mathrm{H}$ peut être plus ou moins abaissé selon le contexte, mais en dehors des tons $\mathrm{H}$ en contexte $\mathrm{H}$... B _ \#\# (cf. section 3.2), il ne se confond généralement pas avec un ton bas, sauf dans des contextes grammaticaux très particuliers. Le ton $\mathrm{H}$ final de telles séquences est en tout cas très nettement perçu

\footnotetext{
${ }^{3}$ Sur la notion de pied appliquée à la phonologie du bambara, cf. entre autres (Green
} 2015). 
comme $\mathrm{H}$ dans des contextes où aucun ton haut n'apparaît en bambara. Il en va de même pour la modulation montante des monosyllabes dont la réalisation alterne en bambara entre $\mathrm{B}$ et $\widehat{\mathrm{BH}}$. Il suffit pour cela que les syllabes concernées ne soient précédées d'aucun ton $\mathrm{H}$ dans les limites du groupe prosodique. Sur ce point très précis, la situation du mandinka du Pakaawu ressemble beaucoup plus à celle du soninké du Kingi décrit dans (Creissels 2016) qu'à celle du bambara standard.

Par exemple en (4), le ton H de bàtú 'entendre' ainsi que la modulation montante de săy 'acheter' sont très nettement perçus.

(4) a. $\grave{A}$ săy!

3SG acheter

'Achète ça!'

b. À bàtú!

3SG attendre

'Attends-le/la!'

Cette question est reprise de façon détaillée à la section 3 .

2.2.3.3. L'alternance $\mathrm{H} \mathrm{B} \sim \mathrm{H} \mathrm{H}$ en fin de mot

Le mandinka du Pakawu a un phénomène de sandhi tonal qui n'a aucun équivalent en bambara standard, maninka-mori ou maninka de Kita. Ce phénomène de sandhi se traduit par une alternance systématique entre séquences tonales $\mathrm{H} \mathrm{B}$ et $\mathrm{H} \mathrm{H}$ en fin de mot, dans des mots qui dans les parlers de référence ont de manière stable une tonalité $\mathrm{H} \mathrm{H}$, comme kilì kilí 'appeler' en (5). Ce point sera repris en détail à la section 4 .

(5) a. À kílì Fántà yé!

3SG appeler Fanta pour

'Appelle-le/la pour Fanta!'

b. À kilí Fàatú yè!

3SG appeler Fatou pour

'Appelle-le/la pour Fatou !'

\subsubsection{Le pont tonal}

Le mandinka du Pakaawu atteste aussi dans plusieurs contextes un type de loi tonale qui à ma connaissance n'a jamais été signalé en mandingue, qui peut se schématiser de la façon suivante :

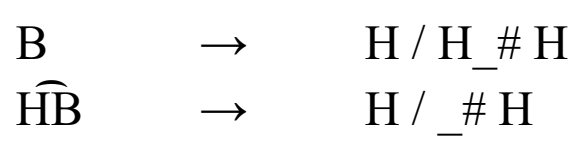

Ce processus, qui sera désigné dans ce qui suit comme PONT TONAL, convertit une séquence tonale $\mathrm{HB} \mathrm{H}$ en $\mathrm{H} \mathrm{H} \mathrm{H}$, et une séquence $\widehat{\mathrm{HB}} \mathrm{H}$ en $\mathrm{H} \mathrm{H}$. A la différence de 
ceux examinés en 2.2.3, il est conditionné par la nature des unités concernées, et parfois aussi par leur relation syntaxique.

La règle de pont tonal s'applique systématiquement chaque fois qu'un pronom personnel non emphatique à ton $\mathrm{H}$ ( $\dot{\eta}$ 'moi' ou $i$ 'toi') est immédiatement précédé d'une séquence tonale $\mathrm{H} \mathrm{B}$ ou d'un ton $\widehat{\mathrm{HB}}$, dans des contextes où par contre on n'observe rien de semblable si on remplace le pronom par un autre mot à ton initial $\mathrm{H}$.

Par exemple, nous pouvons voir en (6a) que devant un mot à ton initial haut, la forme accomplie du verbe 'faire' est normalement réalisée ké-tà. En (6b), la présence de $\dot{\eta}$ 'moi' dans la même position fait apparaître la réalisation $k e ́$-tá, sans qu'aucun abaissement ne vienne compenser la disparition du ton B.

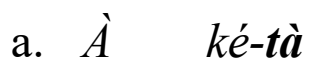
Músáa búlù.
3SG faire-ACP.INTR Moussa SPHP
'Moussa n'a pas fait exprès de faire ça.'
b. $\dot{A}$ ké-tá $\dot{y} \quad$ búlù.
3SG faire-ACP.INTR $1 \mathrm{SG}$ SPHP
'Je n'ai pas fait exprès de faire ça.'

De même en (7a), le prédicatif verbal yè manifeste le ton bas qu'il a normalement

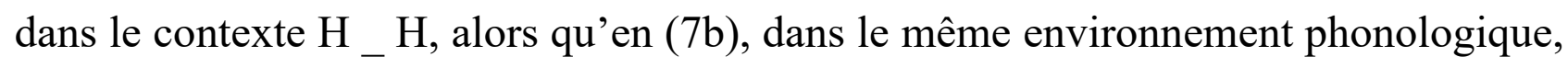
la présence du pronom $\dot{\eta}$ déclenche la règle de pont tonal.
a. Músáa yè
Yáayàa máakóyì.
Moussa ACP.TR Yaya aider
'Moussa a aidé Yaya.'
b. Músáa yé ý máakóyì.
Moussa ACP.TR 1SG aider
'Moussa m'a aidé.'

De manière semblable, en (8a), nîy 'et, avec' manifeste son ton de base $\widehat{\mathrm{HB}}$, tandis qu'en (8b), nîy (dont la nasale finale est élidée) est réalisé $\mathrm{H}$, et aucun abaissement ne vient compenser la disparition du ton $\mathrm{B}$ de sa deuxième more.
a. Yáyàa
nîy Músáa máy
běy.
Yaya et Moussa ACP.NEG s'entendre
'Yaya ne s'entend pas bien avec Moussa.'

b. म́ ní(y) $i$ máy bèy.

$1 \mathrm{SG}$ et 2SG ACP.NEG s'entendre

'Je ne m'entends pas bien avec toi.'

Nous verrons en 7.2.3 qu'une règle de pont tonal opère aussi dans certaines constructions 'nom + modifieur'. 
2.2.5. Différences entre les schèmes tonals possibles pour les lexèmes nominaux et les lexèmes verbaux

Comme dans les autres parlers mandingues dont le système tonal est documenté, les schèmes tonals attestés pour les lexèmes verbaux en mandinka du Pakaawu sont moins nombreux que pour les lexèmes nominaux. Mais en outre, les schèmes tonals possibles pour les lexèmes verbaux ne coïncident avec ceux possibles pour les lexèmes nominaux que lorsque la syllabe finale du lexème est une syllabe légère. Avec les lexèmes à syllabe finale lourde, il y a une différence systématique entre les schèmes tonals des lexèmes nominaux et ceux des lexèmes verbaux. Ceci a pour effet de mettre en défaut la règle selon laquelle, en mandinka, les lexèmes verbaux peuvent s'employer comme noms d'action sans aucune modification formelle (Creissels \& Sambou 2013: 85-96). En effet, en mandinka du Pakaawu, la conversion d'un lexème verbal se terminant par une syllabe lourde en nom d'action implique une modification du contour tonal de base.

Par exemple, en (9), nous pouvons voir que dans le même environnement phonologique (immédiatement avant un mot à ton initial $\mathrm{H}$ ), le verbe dérivé de l'adjectif jówù 'mauvais' par adjonction du suffixe d'abstraction apparaît comme jówúyâa, tandis que le nom correspondant apparaît comme jówúyàa à la forme définie, et jówúyáa à la forme indéfinie. ${ }^{4}$
a. İ $y \grave{~}$
ந’ jówúyâa
kínòo
lá.
3PL ACP.TR 1SG méchant.ABSTR nourriture.D POSTP

'Ils m'ont brimé en me privant de nourriture.'

b. Jówúyàa bóoròo mú jàmfàñóolòo lè tí. méchant.ABSTR.D ACP.TR 1SG éloignement.D FOC POSTP 'Le remède à l'inimitié est l'éloignement.'
c. Jówúyáa $m a \hat{y}$
ké.
méchant.ABSTR ACP.NEG faire
'Rien de méchant n'a été fait.'

\subsubsection{Propriétés tonales des mots grammaticaux et affixes}

Les propriétés tonales de beaucoup de mots grammaticaux et affixes en mandinka du Pakaawu ne correspondent pas à ce qui a été décrit ailleurs en mandingue. Par

${ }^{4}$ Les tons observés en (9a) et (9b) sont d'ailleurs ceux donnés en citation isolée lorsqu'on cite le verbe à la forme de l'infinitif (kà jówúyâa ou k'àa jówúyâa selon que le verbe est envisagé en emploi intransitif ou en emploi transitif) et lorsqu'on cite le nom à la forme définie (jówúyàa - en mandinka du Pakaawu comme dans la plupart des parlers mandingues, c'est la forme définie des noms qui est donnée spontanément par les locuteurs comme forme de citation). 
exemple, la plupart des enclitiques de structure syllabique CV qui manifestent par ailleurs un ton $\mathrm{H}$ prennent en contexte $\mathrm{H}_{-} \mathrm{H}$ un ton bas qui ne correspond à rien de semblable en bambara par exemple. Ceci apparaît à l'exemple (5) ci-dessus avec la postposition yé $\sim$ yè. L'exemple (10) illustre le même phénomène avec la postposition lá $\sim$ là en fonction de génitif.

(10) a. Fántà lá búyò

Fanta GEN chambre.D

'la chambre de Fanta'

b. Fàatú là búnò

Fatou GEN chambre.D

'la chambre de Fatou'

Ce comportement est aussi celui de quelques lexèmes verbaux qui présentent exceptionnellement une structure $\mathrm{CV}$. Cette question sera reprise en détail à la section 6.6.

\subsubsection{Interaction tonale entre le nom et ses modifieurs}

Dans les constructions 'nom + modifieur' où le nom est à la forme du lexème nu (ou forme indéfinie), l'interaction tonale entre nom et modifieur dépend de la nature du modifieur, et les détails de cette interaction sont plus complexes que dans les autres parlers mandingues dont le système tonal est documenté. Ce point sera traité en détail à la section 7.2.

\subsubsection{Propriétés tonales des adverbes idéophoniques}

Contrairement à ce qui a été observé jusqu'ici en mandingue, en mandinka du Pakaawu, les adverbes idéophoniques n'ont pas uniformément un contour tonal entièrement $\mathrm{H}$. Leur contour tonal est toutefois déterminé par leur structure syllabique. Ce point sera traité en 5.4.

\section{Tons hauts faibles}

En mandinka du Pakaawu, un ton $\mathrm{H}$ associé à la syllabe finale d'une unité de deux syllabes ou plus et immédiatement précédé d'un ton bas peut-être qualifié de faible, car selon le contexte il peut subir un abaissement que ne subissent pas les tons $\mathrm{H}$ occupant d'autres positions. Le même phénomène concerne la modulation montante des monosyllabes $\widehat{\mathrm{BH}}$. Toutefois, à la différence d'autres parlers mandingues, on ne peut pas parler d'une véritable alternance entre ton $\mathrm{H}$ et ton $\mathrm{B}$, car en règle générale, l'abaissement des tons $\mathrm{H}$ faibles ne va pas jusqu'à les confondre avec des tons $\mathrm{B}$.

\subsection{Tons hauts faibles immédiatement suivis d'un ton bas}

Dans ce contexte, les tons hauts faibles ne subissent aucun abaissement. 


\subsection{Tons hauts faibles immédiatement suivis d'un ton haut}

Dans ce contexte, les tons hauts faibles subissent un abaissement qui est plus ou moins marqué selon la rapidité du débit, mais en règle générale, leur réalisation ne se confond pas avec celle d'un ton B. Par exemple, le ton $\mathrm{H}$ final d'un lexème comme mùsú 'femme' peut comme en (11a) être véritablement remplacé par un ton B du fait de la loi de compacité tonale qui opère dans les composés. Par contre en (11b), l'abaissement qu'il subit (indiqué ici par le symbole phonétique du ton moyen) est variable selon la rapidité du débit, mais ne va généralement pas jusqu'à la confusion avec un ton $\mathrm{B}$.

(11) a. mùsù-náakòo

femme-jardin.D

'jardin de femme'

b. mùsú náanì [mùsū ná:nì]

femme quatre

'quatre femmes

\subsection{Tons hauts faibles en fin de groupe prosodique}

De manière générale, en mandinka du Pakaawu, le downdrift est particulièrement marqué en fin de groupe prosodique. Dans ce contexte, l'abaissement des tons hauts faibles est particulièrement sensible : à la seule condition qu'au moins un autre ton $\mathrm{H}$ figure dans les limites du même groupe prosodique, un ton $\mathrm{H}$ précédé d'un ton $\mathrm{B}$ et immédiatement suivi de pause est abaissé au même niveau que le ton $\mathrm{B}$ auquel il succède. Par contre, un ton $\mathrm{H}$ précédé d'un ton $\mathrm{B}$ et immédiatement suivi de pause reste nettement perçu comme $\mathrm{H}$ si aucun ton $\mathrm{H}$ ne le précède dans les limites du groupe prosodique. On peut comparer par exemple :
a. ’̀ nàatí!
3SG apporter
'Apporte ça!'
vs. Jíyóo nàatì!
eau.D apporter

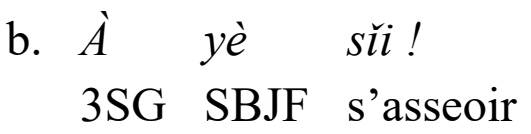
vs. À kánáa sì !
'Qu'il s'asseoie !'
3SG PROH s'asseoir
'Qu'il ne s'assoie pas !

\subsection{Conclusion}

Les observations présentées en 3.2 et 3.3 montrent que la seule analyse qui ne soulève aucune difficulté est qu'en mandinka du Pakaawu, les syllabes (ou mores, dans le cas des monosyllabes) qui présentent ce type d'alternance sont structurellement associées à un ton haut soumis à un phénomène d'abaissement conditionné par le contexte, et qui peut se trouver accentué par l'effet de downdrift. Pour d'autres variétés 
mandingues, on a pu proposer des analyses selon lesquelles ces tons hauts ne figurent pas tels quels dans la structure sous-jacente et sont introduits par une règle d'insertion tenant compte de la structure en pieds, ou s'expliquent par des limitations à la propagation des tons $\mathrm{B}$ dans des systèmes à ton $\mathrm{B}$ marqué. ${ }^{5}$ Par contre en mandinka du Pakaawu, un tel traitement serait difficilement compatible avec le fait que (a) l'abaissement d'un ton haut faible suivi d'un autre $\mathrm{H}$ ne va généralement pas jusqu'à la confusion avec un ton $\mathrm{B}$, et (b) un ton $\mathrm{H}$ faible devant pause ne peut se confondre avec un ton bas que s'il est précédé d'un autre ton $\mathrm{H}$ dans les limites du groupe prosodique.

L'abaissement des tons hauts faibles étant à la fois prédictible en fonction du contexte et variable selon la rapidité du débit, il ne sera pas noté dans la suite de cet article, mais le lecteur est invité à se souvenir du cas limite d'un ton $\mathrm{H}$ en contexte B_\#\#, qui n'est réellement perceptible comme $\mathrm{H}$ que si aucun autre $\mathrm{H}$ ne le précède dans les limites du groupe prosodique.

\section{L'alternance $H \mathbf{H} \sim \mathrm{H} B$ en fin de mot et le relèvement tonal}

Une propriété remarquable du Mandinka du Pakaawu est qu'il est très courant dans ce parler d'observer des mots qui se terminent par une séquence tonale ... H B dans des contextes où, $d$ 'après ce qui s'observe généralement en mandingue, on attendrait plutôt une séquence ... H H. En voici quelques exemples :
a. $\dot{A}$ sì fúntì búnò kónò.
2SG POT sortir chambre.D dans
'Il pourrait sortir de la chambre.'
b. ஜ́ mày súykútù jé jěe.
1SG ACP.NEG fille voir là
'Je n'ai vu aucune fille là-bas.'
c. Yírì náaní bòyítà lè. arbre quatre tomber.ACP.INTR FOC 'Quatre arbres sont tombés.

L'observation cruciale pour l'analyse de ce phénomène est que, quelle que soit la nature morphosyntaxique du mot, et quelle que puisse être la nature morphologique ou phonologique de sa syllabe finale, il n'existe en Mandinka du Pakaawu aucun mot qui se terminerait par une séquence tonale ... H B en tous contextes.

En mandinka du Pakaawu, sans aucune exception, les mots qu'on peut relever dans certains contextes avec une tonalité ... H B prennent une tonalité ... H H à la seule

${ }^{5}$ Le maninka de Kita est un bon exemple de parler se prêtant parfaitement à ce type d'analyse, cf. (Creissels 2009; Creissels \& Grégoire 1993). 
condition d'être immédiatement suivis d'un mot à ton initial bas autre que le focalisateur lè ou le prédicatif verbal yè. Par exemple :
a. $\grave{A}$ sì fúntí dìnkôo kónò.
2SG POT sortir trou.D dans
'Il pourrait sortir du trou.'

b. ஜ́ mày súykútú tàrá jěe.

1SG ACP.NEG fille rencontrer là

'Je n'ai rencontré aucune fille là-bas.'

c. Yírí fùlá bòyítà lè.

arbre deux tomber.ACP.INTR FOC

'Deux arbres sont tombés.

On peut faire exactement les mêmes observations avec les mots monosyllabiques relevés dans certains contextes au moins avec un ton $\widehat{\mathrm{HB}}$, ainsi qu'avec les mots de deux syllabes ou plus relevés dans certains contextes au moins avec un ton final $\widehat{\mathrm{HB}}$. Comme cela est illustré en (15) et (16), si dans le même environnement morphosyntaxique on les fait suivre immédiatement d'un mot à ton initial $\mathrm{B}$ autre que le focalisateur lè ou le prédicatif verbal yè, le ton $\widehat{\mathrm{HB}}$ laisse automatiquement la place à un ton $\mathrm{H}$.
a. Sûu náaní jànítà
lè.
maison quatre brûler.ACP.INTR FOC
'Quatre maisons ont brûlé.'
b. Súu fùlá jànítà
lè.
maison deux brûler.ACP.INTR FOC
'Deux maisons ont brûlé.'
(16) a. Músáa mày féy dádâa Báakàrí yè.
Moussa ACP.NEG chose réparer Bakari pour
'Moussa n'a rien réparé pour Bakari.'
b. Músáa mày féy dádáa Tùmáani yé.
Moussa ACP.NEG chose réparer Toumani pour
'Moussa n'a rien réparé pour Toumani.'

Dans tous les cas, comme cela est illustré en (17) et (18), si le mot en question est susceptible d'être suivi de pause, la forme qui se retrouve devant pause est toujours celle des deux qui se termine par un ton $\mathrm{B}$ ou $\widehat{\mathrm{HB}}$.
a. Ñàykúmòo bé chat.D COPLOC chaise.D sous sìiráyó dùumá.
'Le chat est sous la chaise.' 
b. Ñàykúmóo jòlôn-tá năy yíròo sántò. chat.D tomber-ACP.INTR VEN arbre.D en.haut

'Le chat est tombé du haut de l'arbre.'

c. $\dot{A}$ bé kó ñàykúmòo.

3SG COPLOC comme chat.D

'Ça ressemble à un chat.'
$\begin{array}{lllll}\text { a. } & \grave{A} & \text { sì } & \text { fúntì búyò } & \text { kónò. } \\ \text { 2SG } & \text { POT } & \text { sortir } & \text { chambre.D dans }\end{array}$
'Il pourrait sortir de la chambre.'
b. ’̀ sì fúntí dìnkôo kónò.
2SG POT sortir trou.D dans
'Il pourrait sortir du trou.'
c. $\grave{A}$ sì fúntì.
2SG POT sortir
'Il pourrait sortir.'

Ce type d'alternance tonale à la finale des mots est omniprésent en mandinka du Pakaawu, et à la différence du phénomène évoqué à la section 3, il s'agit clairement d'une alternance entre ton $\mathrm{B}$ et ton $\mathrm{H}$ (ou entre ton $\widehat{\mathrm{HB}}$ et ton $\mathrm{H}$ ), en aucun cas d'une modification qui affecterait la réalisation précise d'un ton sans en changer vraiment l'identité.

La question est donc de savoir, lorsqu'une telle alternance se présente, si le ton B ou $\widehat{\mathrm{HB}}$ qui apparaissent à la finale d'un mot lorsque le mot suivant commence par ton $\mathrm{H}$ (et aussi éventuellement devant pause) appartient à la structure tonale du mot, ou s'il peut être analysé d'une manière ou d'une autre comme introduit par une règle dont l'application dépendrait du contexte.

L'observation cruciale est que, si on choisit d'analyser le ton B qui apparait dans les contextes $\mathrm{H}_{-} \# \mathrm{H}$ et $\mathrm{H}_{\text {_ }} \# \#$ comme n'appartenant pas à la structure tonale du mot et introduit par une règle, il sera impossible de rendre compte de façon simple du fait qu'on peut trouver dans les mêmes contextes des mots se terminant invariablement par une séquence H H. Par exemple, le prénom Yáayàa Yáayáa fait partie des mots qui présentent l'alternance tonale analysée à cette section, tandis que Músáa a invariablement (c'est-à-dire, y compris devant ton $\mathrm{H}$ ou devant pause) le contour tonal H H.

$$
\begin{aligned}
& \text { a. Í mày Yáayàa jé jěe. } \\
& \text { 1SG ACP.NEG Yaya voir là } \\
& \text { 'Je n'ai pas vu Yaya là-bas.' }
\end{aligned}
$$


b. ஜ́ mày Yáayáa tàrá jěe.

1SG ACP.NEG Yaya trouver là

'Je n'ai pas trouvé Yaya là-bas.'

c. ’́ mày Músáa jé jěe.

1SG ACP.NEG Moussa voir là

'Je n'ai pas vu Moussa là-bas.'

d. ந́ mày Músáa tàrá jěe.

1SG ACP.NEG Moussa trouver là

'Je n'ai pas trouvé Moussa là-bas.'

On voit mal comment rendre compte de la réalisation Yáayàa devant ton $\mathrm{H}$ ou pause par une règle introduisant d'une manière ou d'une autre un ton B sans que cette règle fasse une prédiction incorrecte dans le cas de Músáa. Or des mots présentant quel que soit le contexte un contour tonal ... H H se rencontrent dans différentes catégories morphosyntaxiques. Outre quelques noms propres de personnes comme Músáa, on peut citer par exemple les termes de parenté inusités à la forme définie, comme báamáa 'mère', les particules fánáa 'aussi' et dóróy 'seulement', etc. (cf. à la section 5.3 le tableau des séquences tonales possibles à la finale des mots dissyllabiques). Donc, la seule solution simple (et qui d'ailleurs n'entraîne aucune difficulté pour la suite de l'analyse) consiste à poser que dans l'alternance ... H B $\sim \ldots \mathrm{H} \mathrm{H}$ ou $\ldots \widehat{\mathrm{HB}} \sim \ldots \mathrm{H}$ analysée à cette section, c'est le contour tonal... H B ou ... $\widehat{\mathrm{HB}}$ qui est basique, le contour ... H H ou ... H résultant d'une règle de RELÈVEMENT TONAL qu'on peut schématiser comme suit, et qui a pour conséquence de neutraliser une distinction observable dans les contextes où cette règle n'opère pas : ${ }^{6}$

$$
\begin{aligned}
& \mathrm{B} \rightarrow \mathrm{H} / \mathrm{H}_{-} \# \mathrm{~B} \\
& \widehat{\mathrm{HB}} \rightarrow \mathrm{H} /_{-} \# \mathrm{~B}
\end{aligned}
$$

Il faut simplement préciser qu'il existe deux exceptions au conditionnement général de cette règle de relèvement tonal (pour lesquelles je n'ai aucune explication à proposer) : comme cela a déjà été mentionné, le contour tonal ... H B ou ... HB est maintenu si le mot suivant est le prédicatif verbal yè (accompli transitif ou subjonctif) ou le focalisateur lè. Par exemple, en (20a), la règle de relèvement tonal s'applique à Bíntù, alors qu'elle ne s'applique pas en (20b-c).
a. Bíntú kà tábíròo ké.
Bintou INACP cuisine.D faire
'Bintou fait habituellement la cuisine.'

${ }^{6}$ Cette règle est semblable à la règle de 'tonal raising' du Susu, cf. (Green, Anderson \& Obeng 2013). 
b. Bíntù lè kà tábíròo ké.

Bintou FOC INACP cuisine.D faire

'C'est Bintou qui fait habituellement la cuisine.'

c. Bíntù yè tábíròo ké.

Bintou ACP.TR cuisine.D faire

'Bintou a fait la cuisine.'

\section{Ton et poids syllabique, et contraintes sur les séquences tonales dans les limites du mot}

\subsection{Tons complexes et poids syllabique}

En mandinka du Pakaawu, il n'existe aucune exception à la règle selon laquelle les tons modulés $\widehat{\mathrm{HB}}$ et $\widehat{\mathrm{BH}}$ sont possibles seulement sur des syllabes lourdes $\mathrm{CVV}$ ou CVN ou supra-lourdes CVVN. En outre, le ton doublement modulé $\widehat{\mathrm{BHB}}$ est possible seulement sur les syllabes CVV dont la voyelle est $a a$, ee ou oo, et sur les syllabes supra-lourdes (CVVN). A l'exception du nom propre Làây (forme contractée de Làamíni), le ton $\widehat{\mathrm{BHB}}$ résulte toujours du rattachement du ton bas de la marque de défini (devenu flottant du fait de l'effacement de son support segmental) à un lexème nominal de structure $\mathrm{C} \breve{V} \mathrm{~V}$.

Cette distribution suggère de considérer que l'unité porteuse de ton est la more, les syllabes lourdes étant constituées de deux mores et les syllabes supra-lourdes de trois mores. Toutefois, l'intérêt de cette analyse est limité par le fait qu'à différents égards, le comportement tonal d'une syllabe lourde est loin d'être totalement assimilable à celui d'une séquence de deux syllabes légères, ce qui veut dire que dans une formalisation ayant recours systématiquement à la notion de more, il faudrait prévoir que deux mores appartenant à la même syllabe puissent ne pas toujours se comporter comme deux mores constituant une syllabe chacune.

\subsection{Tons complexes dans les mots de deux syllabes ou plus}

On observe en mandinka du Pakaawu des restrictions particulières à l'occurrence des tons complexes dans les limites de mots de deux syllabes ou plus :

- la SYLLABE FINALE d'un mot de deux syllabes ou plus peut si elle est lourde porter un ton $\widehat{\mathrm{HB}}$, sans restriction d'ordre phonologique quant à la nature tonale du ton précédent (cf . par exemple dádâa 'réparer', bàsôo 'natte.D'), par contre elle ne peut en aucun cas porter un ton $\widehat{\mathrm{BH}}$;

- une SYLLABE NON FINALE d'un mot de deux syllabes ou plus ne peut porter un ton complexe $(\widehat{\mathrm{HB}}$ ou $\widehat{\mathrm{BH}})$ que si sa deuxième more porte un ton opposé au ton suivant, et si sa première more porte un ton opposé au ton précédent (où cas où la syllabe en question n'est pas en position initiale). 
En fait, en mandinka du Pakaawu, il existe un certain nombre de mots dissyllabiques à première syllabe lourde avec un ton complexe sur leur première syllabe (comme yăarà 'apparemment' ou têemá 'entre'), par contre il est extrêmement rare de trouver des tons complexes sur les syllabes non finales de mots de deux syllabes ou plus. On peut toutefois citer quelques noms comme kùnlîimáaròo 'l'arc-en-ciel' ou săakùlâa 'le saakulaa (sorte de héron)'.

\subsection{Séquences tonales possibles pour les deux dernières syllabes de mots de deux syllabes ou plus}

Compte tenu du fait que l'analyse des alternances tonales en fin de mot est un point essentiel dans la description tonologique du mandinka du Pakaawu, il est particulièrement important de préciser ici les contraintes qui limitent les séquences tonales possibles sur les deux dernières syllabes des mots de deux syllabes ou plus.

Si on met à part le cas particulier de mots se terminant par le marqueur de pluriel -lú, qui sera discuté en 6.3, on peut poser comme règle que les mots de deux syllabes ou plus dont la syllabe finale est une syllabe légère ne peuvent pas avoir de façon stable un schème tonal ... H H. De tels mots peuvent seulement avoir une alternance ... H H ... H B avec la distribution qui a été analysée à la section 4. Selon l'analyse proposée, cela veut dire que ... H H est impossible comme contour tonal de base d'un mot dont la syllabe finale est une syllabe légère, et plus généralement que le ton de base d'une syllabe légère en fin de mot est prédictible d'après le ton de la syllabe pénultième. ${ }^{7}$

Comme on peut le voir d'après le tableau 1 , qui donne la totalité des possibilités tonales pour les deux dernières syllabes des mots (abstraction faite de possibles modifications dues au relèvement tonal), il n'y a pas de restriction comparable pour les mots dont la syllabe finale est une syllabe lourde.

Etant donné que les noms communs et les verbes sont soumis à des contraintes supplémentaires (différentes d'ailleurs pour les noms et pour les verbes) qui seront analysées en détail aux sections 7 et 8, toutes les illustrations données dans le tableau 1 proviennent d'autres catégories morphosyntaxiques.

${ }^{7}$ La seule exception potentielle à cette généralisation est la forme non-emphatique du pronom de deuxième personne du pluriel álí, dont la réalisation tonale est invariablement H H. Mais on peut estimer qu'il ne s'agit pas d'une véritable exception, pour les deux raisons suivantes. La première est qu'il s'agit d'un proclitique. La deuxième est que ce comportement exceptionnel est certainement lié au fait que, même si synchroniquement álí n'est pas segmentable, sa deuxième syllabe est probablement apparentée au morphème de pluriel lú, qui a des propriétés tonales particulières - cf. 6.3 . 
Tableau 1. Séquences tonales possibles pour les deux dernières syllabes des mots

\begin{tabular}{|c|c|c|c|}
\hline $\begin{array}{l}\text { Ton de la } \\
\text { syllabe } \\
\text { pénultième }\end{array}$ & $\begin{array}{l}\text { Poids de la } \\
\text { syllabe finale }\end{array}$ & $\begin{array}{l}\text { Tons possibles } \\
\text { pour la syllabe } \\
\text { finale }\end{array}$ & Exemples \\
\hline $\mathrm{H}$ ou $\widehat{\mathrm{BH}}$ & légère & $\mathrm{B}$ & $\begin{array}{l}\text { búkà (prédicatif d'inaccompli) } \\
\text { ntè 'moi' } \\
\text { hánì 'même' } \\
\text { báakè 'très' } \\
\text { náanì 'quatre' } \\
\text { kònóntò 'neuf' } \\
\text { dǔumà 'par terre' } \\
\text { yăarà 'apparemment' } \\
\text { wǒotò 'là' } \\
\text { Sěejò 'Sédhiou' } \\
\text { sántò 'en haut' } \\
\text { kámmà 'pour' } \\
\text { Fántà (nom propre) } \\
\text { Mámád ̀̀ (nom propre) } \\
\text { Kèekútà (nom propre) }\end{array}$ \\
\hline $\mathrm{B}$ ou $\widehat{\mathrm{HB}}$ & légère & $\mathrm{H}$ & $\begin{array}{l}\text { ǹté 'ce n'est pas' } \\
\text { àté 'lui / elle' } \\
\text { ñtèlú 'nous' } \\
\text { têemá 'entre' } \\
\text { bétèké 'bien' } \\
\text { wórówùlá 'sept' } \\
\text { Sùntú (nom propre) } \\
\text { Kìsimá (nom propre) } \\
\text { Báakàrí (nom propre) } \\
\text { Lăasàná (nom propre) }\end{array}$ \\
\hline $\mathrm{H}$ ou $\widehat{\mathrm{BH}}$ & lourde & B & $\begin{array}{l}\text { kánàa (prédicatif de } \\
\text { prohibitif) } \\
\text { kúnùy 'hier' } \\
\text { sáyìy 'maintenant' } \\
\text { dántày 'quelques' } \\
\text { nàmánày 'avant que' } \\
\text { Sírèe (nom propre) } \\
\text { Fárìy (nom propre) }\end{array}$ \\
\hline
\end{tabular}




\begin{tabular}{|c|c|c|c|}
\hline $\begin{array}{l}\text { Ton de la } \\
\text { syllabe } \\
\text { pénultième }\end{array}$ & $\begin{array}{l}\text { Poids de la } \\
\text { syllabe finale }\end{array}$ & $\begin{array}{l}\text { Tons possibles } \\
\text { pour la syllabe } \\
\text { finale }\end{array}$ & Exemples \\
\hline & & $\mathrm{H}$ & $\begin{array}{l}\text { fánáy fánáa 'aussi' } \\
\text { dóróy 'seulement' } \\
\text { síníy 'demain' } \\
\text { fólóo 'd'abord' } \\
\text { túyáa 'à l'étranger' } \\
\text { Músáa (nom propre) } \\
\text { Álánsóo (nom propre) } \\
\text { bírîy 'depuis' kàbírîy }\end{array}$ \\
\hline B ou HB & lourde & $\begin{array}{l}\mathrm{H} \\
\widehat{\mathrm{HB}}\end{array}$ & $\begin{array}{l}\text { mùntóo 'où' } \\
\text { jìmáa 'qui ?' } \\
\text { Nàndíy (nom propre) } \\
\text { Màanifáa (nom propre) } \\
\text { mùwây 'vingt' } \\
\text { wòtôo 'donc' } \\
\text { ìyôo 'd'accord!' } \\
\text { sânsây 'immédiatement' } \\
\text { Màalây (nom propre) } \\
\text { Hàwâa (nom propre) }\end{array}$ \\
\hline
\end{tabular}

\subsection{Le cas particulier des adverbes idéophoniques}

Sans aucune exception, les adverbes idéophoniques du Mandinka du Pakaawu que j'ai relevés se terminent pas une syllabe lourde ou supra-lourde, et à une seule exception près (mii, associé au verbe fī 'être/devenir noir'), leur syllabe finale est de type $\mathrm{CV}(\mathrm{V}) \mathrm{C}$. Contrairement aux autres types de mots, pour lesquels la seule consonne possible en position finale est une nasale qui devant pause se réalise $\eta$, les adverbes idéophoniques peuvent avoir comme consonne finale l'une des consonnes suivantes : $p, t, c, k, b, j, f, s, m, \eta, r, w$ ou $y$.

En ce qui concerne leur ton, il n'est pas le même pour tous les adverbes idéophoniques, mais il est néanmoins déterminé par la structure syllabique: les adverbes idéophoniques se terminant par $m, r$ ou $w$ ont un ton $\widehat{\mathrm{HB}}$ sur leur syllabe finale (et un ton invariablement $\mathrm{H}$ sur les syllabes précédentes, s'ils comportent plus d'une syllabe : párâw, pirîm, etc.), alors que ceux terminés par une consonne autre que $m, r$ ou $w$ ont une tonalité entièrement haute (páfát, kúdéy, etc.).

On remarque que les consonnes qui appellent un ton descendant sur la syllabe finale des adverbes idéophoniques ne constituent pas une classe naturelle (pas plus 
d'ailleurs que l'ensemble des consonnes susceptibles d'apparaître à la finale des adverbes idéophoniques).

\section{Particularités tonales des mots monosyllabiques}

\subsection{Monosyllabes lourds ou supra-lourds}

Les mots monosyllabiques constitués d'une syllabe lourde ou supra-lourde peuvent avoir comme ton de base $\mathrm{H}, \widehat{\mathrm{BH}}, \widehat{\mathrm{HB}}$ ou $\widehat{\mathrm{BHB}}$.

En dehors du prénom masculin $L a \grave{a} y$ (forme contractée de Làamíni), le ton $\widehat{\mathrm{BHB}}$ est exclusivement attesté par la forme définie de noms monosyllabiques dont la voyelle est aa (bàâ 'la chèvre), ee (kèe 'l'homme') ou oo (kòô 'le sel'). L'explication est que de telles formes cumulent le ton lexical $\widehat{\mathrm{BH}}$ et le ton bas du suffixe $-\dot{o}$.

Phonologiquement, les tons $\mathrm{H}, \widehat{\mathrm{BH}}$ et $\widehat{\mathrm{HB}}$ sont également courants pour les mots monosyllabiques constitués d'une syllabe lourde, mais comme cela sera discuté en détail aux sections 7 et 8 , leur distribution précise est conditionnée par la nature grammaticale des mots. Outre les formes nominales et verbales qui seront discutées en 7 et 8 , on peut illustrer la variété tonale des mots monosyllabiques constitués d'une syllabe lourde par les exemples suivants :

- monosyllabes à ton $\mathrm{H}$ : le déterminant ou pronom indéfini dóo ;

- monosyllabes à ton $\widehat{\mathrm{BH}}$ : les démonstratifs ñ̌y et wǒo, le marqueur de passé nǔy,

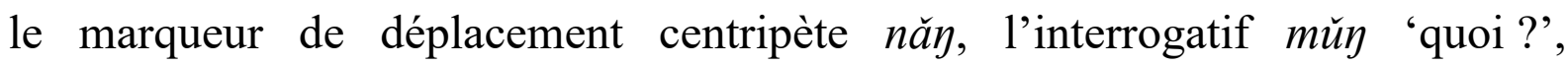
l'interrogatif lěe 'où se trouve ... ?', etc. ${ }^{8}$

- monosyllabes à ton $\widehat{\mathrm{HB}}$ : le totalisateur bêe 'tout, tous', le relativiseur mîy, le prédicatif d'accompli négatif $m a ̂ y$, la préposition comitative nîy 'avec', le coordinatif nî̀ 'et', la conjonction $n \hat{\imath} \eta$ 'si', les postpositions kây et fêe, le numéral tây 'dix', etc.

Comme cela est illustré en (21), les mots monosyllabiques constitués d'une syllabe lourde à ton $\widehat{\mathrm{HB}}$ sont soumis à une règle tonale spéciale qui remplace leur ton $\widehat{\mathrm{HB}}$ par

${ }^{8}$ Quelques-uns des monosyllabes Č̌V ont dans certains contextes une variante $C \dot{V}$ ou $\mathrm{CV}$ dont l'apparition ne relève d'aucune régularité et ne se prête à aucune généralisation :

- le démonstratif wǒo a une variante wó lorsqu'il est immédiatement suivi du focalisateur

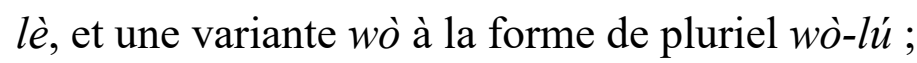

- le démonstratif ñ̆y a une variante ñí en combinaison avec le focalisateur lè (qui se nasalise en nè malgré l'élision de la nasale finale de nĭy : ní nè), et une variante ñì à la forme de pluriel ñì-lú.

- l'interrogatif mŭy a une variante mú en combinaison avec le focalisateur lè (qui se nasalise en nè malgré l'élision de la nasale finale de mǔy : mú nè). 
un ton B s'ils sont immédiatement précédés par un mot à ton final $\mathrm{H}$ dont aucune pause ne les sépare et immédiatement suivis par une pause ou un mot à ton initial $\mathrm{H}$ : $\widehat{\mathrm{HB}} \rightarrow \mathrm{B} / \mathrm{H}_{-} \mathrm{H}_{2} \mathrm{H}_{-} \#$
(21) a. Súnkútù mây fúntì.
fille ACP.NEG sortir
'Aucune fille n'est sortie.'
b. Mùsú mày fúntì.
femme ACP.NEG sortir
'Aucune femme n'est sortie.'

\subsection{Monosyllabes légers : remarques générales}

Compte tenu de l'impossibilité pour les syllabes légères d'être associées à un ton complexe, on s'attendrait a priori à ce que les monosyllabes légers se répartissent en deux classes tonales, H et B. En fait, il n'en est rien, car l'immense majorité des mots ou clitiques constitués d'une syllabe légère ont un ton qui alterne selon une règle qui met en jeu un phénomène de polarité (contraste systématique avec le ton précédent), et qui peut difficilement s'expliquer à partir de structures tonales sous-jacentes où les monosyllabes en question seraient simplement associés à un ton $\mathrm{H}$ ou à un ton $\mathrm{B}$. En outre, les monosyllabes auxquels il convient de reconnaître un ton de base B n'ont pas tous le même comportement combinatoire.

\subsection{Monosyllabes légers à ton invariablement $H$}

Très peu de monosyllabes légers ont un ton invariablement $\mathrm{H}$ en tous contextes. On peut toutefois citer les pronoms personnels non emphatiques (proclitiques) $\dot{\eta}$ (1SG) et $i$ (2SG), la particule énonciative dé, ainsi que le marqueur de pluriel -lú (couramment présenté comme un suffixe dans les descriptions de langues mandingues).

L'exemple (22) montre que le marqueur de pluriel présente invariablement un ton $\mathrm{H}$, y compris en contexte ... $\mathrm{H}_{-} \# \mathrm{H}$. Donc si on l'analyse comme un suffixe, cela introduit des exceptions systématiques à une règle qui par ailleurs ne souffre aucune exception en mandinka du Pakaawu, règle selon laquelle, dans les mots de deux syllabes ou plus à syllabe finale légère, une séquence tonale finale ... H H peut seulement apparaître en alternance avec ... H B.
a. İ fánán-nú táa-tà
kúykòo tó.
3PL aussi-PL aller-ACP.INTR champ.D LOC
'Eux aussi sont partis au champ.'
b. Dóo-lú táa-tà kúykòo tó.
INDEF-PL aller-ACP.INTR champ.D LOC
'Il y en a qui sont partis au champ.'




$\begin{array}{llll}\text { c. } \dot{~} \quad \text { téerímáa-lú táa-tà } & \text { kúnkòo tó. } \\ \text { 1SG ami-PL aller-ACP.INTR } & \text { champ.D } & \text { LOC } \\ \text { 'Mes amis sont partis au champ.' } & \end{array}$

Une façon possible de contourner cette difficulté consiste à poser que le marqueur de pluriel n'est pas véritablement un suffixe, mais plutôt un enclitique, et que les enclitiques ne sont pas pris en considération pour l'évaluation des contraintes sur les séquences tonales en fin de mot.

\subsection{Monosyllabes légers à ton invariablement bas}

Il existe un petit nombre de monosyllabes légers qui ont ton invariablement B en tous contextes : les pronoms personnels non emphatiques (proclitiques) $\grave{a}$ (3SG), $\grave{\eta}$ (1PL) et $i$ (3PL), et le focalisateur (enclitique) lè..$^{9}$

\subsection{Monosyllabes de structure $C V$ à ton variable en réalisation, mais dont le ton de base peut s'analyser comme $B$}

Il existe quelques monosyllabes qui ne peuvent apparaître, ni immédiatement après pause, ni immédiatement avant pause, dont le ton réalisé peut être $\mathrm{H}$ ou $\mathrm{B}$ selon l'environnement, mais avec une distribution qui permet de reconnaitre leur ton de base comme B. En effet, ces enclitiques ne s'observent avec un ton $\mathrm{H}$ que lorsqu'ils sont à la fois immédiatement précédés d'une syllabe à ton $\mathrm{H}$ et immédiatement suivis d'une syllabe à ton $\mathrm{B}$. Ceci est illustré en (23) par le comportement du prédicatif verbal d'inaccompli $k a ̀$, qui apparait comme $k a ́$ en contexte $\mathrm{H}_{-}$B, et comme kà dans tous les autres contextes. On peut rendre compte très simplement de cette distribution en posant un ton structurel $\mathrm{B}$ soumis à la même règle de relèvement tonal que la syllabe finale des mots se terminant par une séquence tonale ... H B :

$\mathrm{B} \rightarrow \mathrm{H} / \mathrm{H}_{-} \# \mathrm{~B}$
a. $\grave{b} \boldsymbol{k} \dot{a}$
tábíròo ké.
1PL INACP cuisine.D faire
'Nous faisons la cuisine.'
b. '́ kà tábíròo ké.
1SG INACP cuisine.D faire
'Je fais la cuisine.'

${ }^{9}$ En mandinka du Pakaawu, la focalisation d'un groupe nominal au pluriel entraîne la répétition de la marque du pluriel après le focalisateur, et l'attachement de la marque du pluriel provoque l'allongement de la voyelle du focalisateur, cf. Kèê lè kúmà-tá 'C'est l'homme qui a parlé' vs. Kèê-lú lèe-lú kúmà-tá 'Ce sont les hommes qui ont parlé'. En débit rapide, la tendance à réduire la voyelle de la première occurrence du marqueur de pluriel peut donner l'impression d'une gémination de la consonne initiale du focalisateur. 
c. ’̀ kì kùurôo ké.

1PL INACP lessive.D faire

'Nous faisons la lessive.'

d. ’́ ká kùurôo ké.

1SG INACP lessive.D faire

'Je fais la lessive.'

La même alternance caractérise aussi le prédicatif verbal yè (accompli transitif et subjonctif), sa variante $\eta \grave{a},{ }^{10}$ et le prédicatif verbal $s \grave{\imath} \sim s \grave{e}$ (potentiel). ${ }^{11}$

\subsection{Monosyllabes de structure $\mathrm{CV}$ à ton variable en réalisation, mais dont le ton de base peut s'analyser comme $\mathrm{Hb}$}

La très grande majorité des monosyllabes légers, lorsqu'ils ne succèdent pas immédiatement à une pause, présentent une alternance entre $\mathrm{H}$ et $\mathrm{B}$ manifestement différente de celle décrite en 6.5, car leur ton est réalisé $\mathrm{H}$ dans la plupart des contextes possibles. Une partie des monosyllabes légers dont le ton varie ainsi sont des enclitiques, ce qui veut dire qu'il est impossible de les trouver immédiatement après une pause, mais ceux parmi eux qui ont cette faculté présentent alors un ton $\mathrm{H}$.

\subsubsection{Lexèmes verbaux dont la forme segmentale alterne entre $C V$ et $C V V$}

De manière générale en mandinka, il existe 6 lexèmes verbaux faisant exception à la règle selon laquelle un lexème nominal ou verbal doit comporter minimalement deux mores : je(e) 'voir' ke(e) 'faire', bo(o) 'sortir', fo(o) 'dire', so(o) 'donner' et tu(u) laisser'. Ces verbes ont un allomorphe CV et un allomorphe CVV. La variante CV est sélectionnée dans les deux cas suivants :

- lorsque le lexème est employé verbalement sans aucun suffixe ;

- lorsque le lexème est employé verbalement avec le suffixe -ta ou avec le suffixe -la.

La variante CVV est sélectionnée dans tous les autres contextes, c'est-à-dire :

- en présence de suffixes autres que -ta ou -la;

- lorsque le lexème est le deuxième formatif de lexèmes complexes ;

- lorsque le lexème est employé nominalement.

La variante $\mathrm{CVV}$ de ces lexèmes verbaux a le ton $\widehat{\mathrm{HB}}$, ce qui est conforme aux correspondances tonales régulières avec les autres parlers mandingues. En cas de

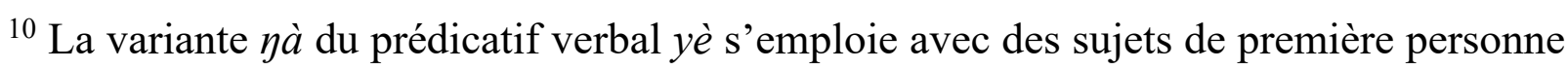
(singulier ou pluriel).

${ }^{11}$ A travers le mandinka, la forme la plus courante du prédicatif 'potentiel' est sì. Dans le mandinka du Pakaawu, la variante dialectale sè est en variation libre avec sì. 
suffixation, la variante $\mathrm{CV}$ présente un ton $\mathrm{H}$ (par exemple, le verbe 'faire' a pour forme d'accompli ké-tà). Enfin, en l'absence de suffixe qui 'stabilise' en quelque sorte son ton, la variante $\mathrm{CV}$ présente l'alternance suivante, qui est tout à fait inattendue d'après le comportement tonal qu'ont ces verbes ailleurs en mandingue:

- Immédiatement après pause, quel que soit le ton initial du mot suivant, le ton de ces verbes est $\mathrm{H}$, comme en (24).
a. Bó búnò kónò!
sortir chambre.D dans

'Sors de la chambre!'
b. Bó wòtôo kónò!
sortir voiture.D dans

'Sors de la voiture !'

- Immédiatement après un mot à ton final $\mathrm{B}$ ou $\widehat{\mathrm{HB}}$, le ton de ces verbes est $\mathrm{H}$, comme en (25).
a. Fántà só kódòo lá!
Fanta donner argent.D POSTP
'Donne l'argent à Fanta!'
b. Fántà só fàanôo lá !
Fanta donner pagne.D POSTP
'Donne le pagne à Fanta!'

- Immédiatement après un mot à ton final $\mathrm{H}$ ou $\widehat{\mathrm{BH}}$, le ton de ces verbes dépend de ce qui suit : $\mathrm{H}$ devant un mot à ton initial $\mathrm{B}, \mathrm{B}$ devant un mot à ton initial $\mathrm{H}$ ou devant pause, comme en (26).
a. Fàatú sò kódòo lá!
Fatou donner argent.D POSTP
'Donne l'argent à Fatou!'
b. Fàatú só fàanôo lá !
Fatu donner pagne.D POSTP
'Donne le pagne à Fatou !'

On peut imaginer plusieurs façons possibles de rendre compte de cette alternance, mais une façon simple de la décrire consiste à poser que :

- le ton de base (ou par défaut) de l'allomorphe $\mathrm{CV}$ de ces verbes est $\mathrm{H}$,

- s'ils ne succèdent pas immédiatement à une pause, leur ton $\mathrm{H}$ est modifié par une règle de polarité qui lui substitue un ton $\mathrm{B}$ à condition que le ton précédent soit $\mathrm{H}$, 
- s'ils ne précèdent pas immédiatement une pause, le résultat de la règle de polarité peut être annulé par la règle de propagation du ton haut $\mathrm{B} \rightarrow \mathrm{H} / \mathrm{H}_{-} \mathrm{B}$.

Une analyse possible est que la tonalité sous-jacente de tels monosyllabes est $\mathrm{Hb}$, avec un ton flottant bas (noté b), qui se substitue au ton $\mathrm{H}$ à condition que celui ne soit précédé ni de pause, ni d'un ton $\mathrm{B}\left(\mathrm{Hb} \rightarrow \mathrm{B} / \mathrm{H}_{-}\right)$, et qui dans le cas contraire est effacé. Ensuite, le ton $\mathrm{B}$ résultant de la substitution est soumis à la règle de propagation du ton $\mathrm{H}\left(\mathrm{B} \rightarrow \mathrm{H} / \mathrm{H}_{-} \mathrm{B}\right)$ lorsqu'il précède immédiatement un autre $\mathrm{B}$.

Je n'ai aucun argument vraiment décisif pour éliminer les autres analyses possibles, mais comme il faut bien disposer d'une notation pour marquer l'appartenance de tels monosyllabes à une classe tonale particulière lorsqu'ils sont cités en isolation, j'utiliserai arbitrairement dans la suite de cet article la notation CV́', sans prétendre que l'analyse qu'elle suggère soit la seule possible, ni même la meilleure.

\subsubsection{Copules}

Les copules mú (copule équative), bé (copule de localisation) et té (copule négative $)^{12}$ présentent la même alternance $\mathrm{H} \sim \mathrm{B}$ avec la même distribution, à ceci près qu'il s'agit d'enclitiques, et qu'il est donc impossible de savoir comment ils pourraient être réalisés en début de groupe prosodique.
a. Fántà bé
búyò
kónò.
Fanta COPLOC chambre.D dans

'Fanta est dans la chambre.'
b. Fántà bé sàatêe tó.
Fanta COPLOC village.D LOC
'Fanta est au village.'
c. Fàatú bè búyò kónò.
Fatou COPLOC chambre.D dans
'Fatou est dans la chambre.'
d. Fàatú bé sàatêe tó.
Fatou COPLOC village.D LOC
'Fatou est au village.'

${ }^{12}$ Il existe une variante ìté de la copule négative, employée exclusivement dans la prédication équative. 


\subsubsection{Postpositions de structure $C V$}

Ce comportement tonal est aussi celui de la totalité des postpositions de structure CV : lá , má ${ }^{13}$ tí, tó ' yé . L'exemple (28) illustre la postposition lá dans son emploi de marque de possession aliénable dans la possession adnominale.
a. Fántà lá búnò
Fanta GEN chambre.D
'la chambre de Fanta'
b. Fántà lá fàanôo
Fanta GEN pagne.D
'le pagne de Fanta.'
c. Fàatú là búyò
Fatou GEN chambre.D
'la chambre de Fatou.'
d. Fàatú lá fàanôo
Fatou GEN pagne.D
'le pagne de Fatou.'

\subsubsection{Le quotatif kó}

L'exemple (29) illustre l'appartenance du quotatif kó à cette classe tonale.
a. Fántà kó kónkòo lè bé à lá.
Fanta QUOT faim.D FOC COPLOC 3SG POSTP
'Fanta dit qu'elle a faim.'
b. Fàatú kò kónkòo lè bé à lá.
Fatou QUOT faim.D FOC COPLOC 3SG POSTP
'Fatou dit qu'elle a faim.'

Il est intéressant d'observer que, si le locuteur marque une pause après le quotatif (ce qui est courant dans le discours spontané), sa voyelle devient longue et le ton devient un ton modulé descendant : kôo.

\section{Le ton dans le système nominal}

\subsection{Classes tonales de lexèmes nominaux et tonalité de la forme définie des noms}

\subsubsection{Remarques introductives}

A l'exception des termes qu'on peut considérer comme assimilés en quelque sorte à des noms propres, comme dúníyáa 'le monde' ou jàhánnàbá 'l'enfer', ainsi que

${ }^{13}$ Contrairement à ce qui s'observe ailleurs en mandingue, il n'y a en mandinka aucune différence de comportement tonal entre lá et $m a ́$ `. 
l'ensemble des noms de relations interpersonnelles lorsqu'ils sont pourvus du suffixe classificateur -máa, comme báa-máa 'mère' ou téerí-máa 'ami' ${ }^{14}$ les noms communs ont une forme définie analysable comme résultant de l'adjonction d'un suffixe $-\grave{o}$.

L'identification de la tonalité de la forme définie des noms communs ne pose aucun problème, car le ton avec lequel cette forme apparaît en citation isolée peut seulement être modifié par la règle générale de relèvement tonal qui convertit ... H B \# en ... H $\mathrm{H} \#$ et ... $\widehat{\mathrm{HB}} \#$ en ... H\#. C'est ce qu'illustre l'exemple (17), repris ici comme (30), avec le nom ñàykúmòo 'chat' $(<$ ñànkúmà $+-o$ ) .
a. Ñàykúmòo bé
sìiráyó dùumá.
chat.D
COPLOC chaise.D sous
'Le chat est sous la chaise.'
b. Ñàykúmóo jòlôn-tá năy yíròo sántò. chat.D tomber.ACP.INTR VEN arbre.D en.haut
'Le chat est tombé du haut de l'arbre.'
c. $\dot{A}$ bé kó ñàykúmòo.
3SG COPLOC comme chat.D
'Ça ressemble à un chat.'

La détermination du contour tonal de base de la forme indéfinie (ou forme nue) est plus problématique, car elle n'est normalement pas citable en isolation, n'apparaît jamais en fin de phrase, et interagit tonalement de façon complexe avec les modifieurs de nom. Dans ces conditions, le choix le plus naturel est de considérer que le contour tonal de base des noms à la forme nue est celui qu'ils manifestent lorsque le nom à la forme nue dépourvu de tout modifieur forme à lui seul une expressions nominale occupant l'une des trois positions $\mathrm{S}, \mathrm{O}$ ou $\mathrm{X}$ dans le schème de prédication verbale $\mathrm{S} \mathrm{p}$ $\mathrm{O} \mathrm{V} \mathrm{X,}{ }^{15}$ dans un contexte phonologique qui ne donne pas lieu à l'application de la règle de relèvement tonal $\mathrm{B} \rightarrow \mathrm{H} / \mathrm{H}_{-} \# \mathrm{~B}$. Ceci est possible sans restriction particulière en contexte négatif, et donc pratiquement, on peut déterminer le contour

${ }^{14}$ En l'absence de ce suffixe -máa propre aux termes de relations interpersonnelles (qu'il convient de bien distinguer de ses homonymes, à savoir le suffixe sélectif -máa des adjectifs et le suffixe propriétif -máa - cf. (Creissels \& Sambou 2013: 115-116)), les mêmes noms ont le comportement normal de noms communs, avec un contraste entre forme indéfinie (báa, téeri) et forme définie (bâa, téeròo). A ce propos, il est intéressant de noter la distinction tonale entre d'une part dímmáa-lú pluriel de dímmáa 'fils ou fille de quelqu'un', et d'autre part dímmàa-lú, pluriel du dérivé propriétif 'personne qui a des enfants' ou díndímmàa-lú 'les plus jeunes', pluriel de la forme sélective de dindin 'enfant' employé adjectivalement.

${ }^{15} \mathrm{~S}=$ sujet, $\mathrm{p}=$ marqueur prédicatif, $\mathrm{O}=$ objet, $\mathrm{V}=$ verbe, $\mathrm{X}=$ oblique. 
tonal de base des lexèmes nominaux en les insérant dans des cadres comme $\mathrm{N}$ tíjăg 'Il n'y a pas de $\mathrm{N}$ ici', $\mathrm{N}$ máy tàrá jěe 'Aucun N n'a été trouvé là-bas').

Dans ce qui suit sont passées en revue, en fonction de la structure syllabique des bases nominales, les manifestations possibles de la présence du marqueur de défini $-o ̀$, notamment au niveau tonal.

De manière générale, il y a dans les parlers mandinka une variation quant à la forme définie des noms dont la base se termine en -ee : ces noms peuvent, de façon variable d'un parler à l'autre, se comporter comme ceux dont la base se termine en - $a a$ ou -oo (avec une manifestation purement tonale de la présence du suffixe - $o$ ), ou bien comme ceux dont la base se termine en $-i i$ ou $-u u$ (avec une manifestation du suffixe $-\grave{o}$ au niveau segmental). Par exemple, la forme définie de sàatée 'village' peut être sàatêe ou sàatéwòo. C'est la première variante qui est prise en considération ici, car c'est celle qu'utilise spontanément le consultant (qui reconnaît toutefois que la deuxième variante n'est pas totalement étrangère à son parler).

\subsubsection{Monosyllabes}

Il n'existe pas de nom dont la base serait constituée d'une syllabe légère. Ceux dont la base est constituée d'une syllabe lourde (CVV et CVN) se répartissent en deux types tonals, $\mathrm{H}$ et $\widehat{\mathrm{BH}}$. Ceux dont la base est constituée d'une syllabe supra-lourde (CVVN) appartiennent tous au type tonal $\widehat{\mathrm{BH}}$.

\subsubsection{Monosyllabes Caa, Cee ou Coo}

L'adjonction du suffixe - $o$ n'a aucune incidence sur la forme segmentale, mais modifie la tonalité d'une façon qui s'explique aisément par le rattachement du ton B de -ò devenu flottant du fait de l'effacement de son support segmental, voir Tableau 2.

Tableau 2. Suffixe $-o$ avec les monosyllabes Caa, Cee ou Coo

\begin{tabular}{|c|c|l|}
\hline Ind. & Def. & Exemples \\
\hline $\mathrm{H}$ & $\widehat{\mathrm{HB}}$ & $b a ́ a / b \hat{a} a$ 'fleuve' \\
\hline$\widehat{\mathrm{BH}}$ & $\widehat{\mathrm{BHB}}$ & $b \check{a} a / b \grave{a} \hat{a}$ 'chèvre' \\
\hline
\end{tabular}

7.1.2.2. Monosyllabes Cuu ou Cii

Tableau 3. Suffixe $-\grave{o}$ avec les monosyllabes Cuu ou Cii

\begin{tabular}{|c|c|l|}
\hline Ind. & Def. & Exemples \\
\hline $\mathrm{H}$ & $\mathrm{H} \mathrm{B}$ & súu / súwòo 'maison' \\
\hline$\widehat{\mathrm{BH}}$ & $\mathrm{B} \widehat{\mathrm{HB}}$ & sǔu / sùwôo 'cheval' \\
\hline
\end{tabular}

Les monosyllabes $\mathrm{Cuu}$ et $\mathrm{Cii}$ ont une forme définie dissyllabique, avec une première voyelle brève et un $o$ long dans la deuxième syllabe $(i i+o \rightarrow$ iyoo, $u u+o \rightarrow$ uwoo). Si le lexème a pour ton de base $\widehat{\mathrm{BH}}$, l'effacement de sa deuxième more à la 
forme définie entraîne le report du ton $\mathrm{H}$ sur la première more de la syllabe suivante $(\widehat{\mathrm{BH}}+\mathrm{B} \rightarrow \mathrm{B} \widehat{\mathrm{HB}})$.

\subsubsection{Monosyllabes CVN}

A la forme définie, la nasale finale est re-syllabifiée comme attaque d'une syllabe dont le noyau est $o$ (si le lexème a $\mathrm{H}$ pour ton de base) ou oo (si le lexème a $\widehat{\mathrm{BH}}$ pour ton de base). Comme dans le cas précédent, si le lexème a pour ton de base $\widehat{\mathrm{BH}}$, la resyllabification qui rend la première syllabe légère a pour conséquence le report du ton $\mathrm{H}$ associé à la deuxième more du lexème sur la première more de la syllabe suivante $(\widehat{\mathrm{BH}}+\mathrm{B} \rightarrow \mathrm{B} \widehat{\mathrm{HB}})$.

Tableau 4. Suffixe $-\grave{o}$ avec les monosyllabes CVN

\begin{tabular}{|c|c|l|}
\hline Ind. & Def. & Exemples \\
\hline $\mathrm{H}$ & $\mathrm{H} \mathrm{B}$ & súy / súyò 'jeûne' \\
\hline$\widehat{\mathrm{BH}}$ & $\mathrm{B} \widehat{\mathrm{HB}}$ & sŭy / sùyôo 'voleur' \\
\hline
\end{tabular}

\subsubsection{Monosyllabes CVVN}

Les monosyllabes CVVN ont tous un ton de base $\widehat{\mathrm{BH}}$. Comme la re-syllabification qu'entraîne l'adjonction de -ò laisse subsister une syllabe lourde, le ton $\widehat{\mathrm{BH}}$ se maintient sur la première syllabe de la forme définie, la deuxième syllabe ayant un $o$ bref à ton B.

Tableau 5. Suffixe $-\grave{o}$ avec les monosyllabes CVVN

\begin{tabular}{|c|c|l|}
\hline Ind. & Def. & Exemples \\
\hline$\widehat{\mathrm{BH}}$ & $\widehat{\mathrm{BH}} \mathrm{B}$ & bǒoy / bǒonò 'bambou' \\
\hline
\end{tabular}

L'existence de syllabes supra-lourdes CVVN est une particularité d'une partie des parlers mandinka. Les syllabes supra-lourdes s'expliquent parfois par la rencontre d'un lexème se terminant par une voyelle longue et d'un suffixe dérivatif commençant par une séquence NC, comme cela est signalé par Creissels \& Sambou (2013: 29) pour le parler de Sédhiou, par exemple báa 'mère' + -ntáy suffixe privatif $\rightarrow$ báantáy 'qui n'a pas de mère). En mandinka du Pakaawu, il existe aussi quelques lexèmes monosyllabiques (tous nominaux, et tous à ton $\widehat{\mathrm{BH}}$ ) qui ont de manière inhérente la

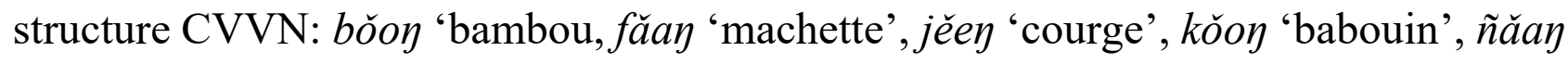

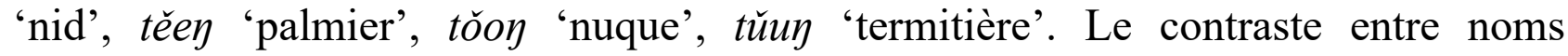
monosyllabiques $\mathrm{CV} \mathrm{V}$ et $\mathrm{C} \breve{V} \mathrm{VN}$ se perçoit de façon particulièrement nette à la forme définie, du fait de la différence de contour tonal (B $\widehat{\mathrm{HB}}$ vs. $\widehat{\mathrm{BH}} \mathrm{B})$.

\subsubsection{Dissyllabes à syllabe finale légère}

Le ton de base de la syllabe finale des dissyllabes à syllabe finale légère est systématiquement l'opposé du ton précédent, ce qui donne deux possibilités pour les 
dissyllabes dont les deux syllabes sont légères, et quatre pour ceux constitués d'une première syllabe lourde et d'une deuxième syllabe légère. L'adjonction de -ò ne modifie pas le ton de la deuxième syllabe lorsque celui-ci est $\mathrm{B}$, et fait apparaître un ton $\widehat{\mathrm{HB}}$ lorsque le ton de base de la syllabe finale est $\mathrm{H}$.

Tableau 6. Suffixe $-o$ avec les dissyllabes à syllabe finale légère

\begin{tabular}{|c|c|l|}
\hline Ind. & Def. & Exemples \\
\hline H B & H B & $\begin{array}{l}\text { básà / básòo 'lézard' } \\
\text { cáabì / cáabòo 'clef' } \\
\text { kúykù / kúykòo 'champ' }\end{array}$ \\
\hline B H & B $\widehat{\mathrm{HB}}$ & $\begin{array}{l}\text { mùsú / mùsôo 'femme' } \\
\text { bòotó / bòotôo 'sac' } \\
\text { bàmbá / bàmbôo 'crocodile' }\end{array}$ \\
\hline BH B & $\widehat{\mathrm{BH}} \mathrm{B}$ & $\begin{array}{l}\text { băanà / băanòo 'arbre, sp.' } \\
\text { kŭnnà / kŭnnòo 'faveur divine' }\end{array}$ \\
\hline$\widehat{\mathrm{HB}} \mathrm{H}$ & $\widehat{\mathrm{HB}} \widehat{\mathrm{HB}}$ & \begin{tabular}{l} 
kôomá / kômôo 'arrière' \\
\hline
\end{tabular}
\end{tabular}

\subsubsection{Dissyllabes à syllabe finale lourde}

Avec les lexèmes nominaux dissyllabiques à syllabe finale lourde, le ton de base de la syllabe finale est invariablement $\mathrm{H}$. D'après les régularités qui ont été dégagées, un ton modulé sur la première syllabe serait possible, mais cette possibilité n'est pas attestée, ce qui laisse deux types tonals attestés sur les quatre théoriquement possibles.

7.1.4.1. Dissyllabes à syllabe finale $\mathrm{Caa}, \mathrm{Cee}$ ou $\mathrm{Coo}$

La deuxième syllabe a à la forme définie un ton $\mathrm{B}$ ou $\widehat{\mathrm{HB}}$ qui contraste avec le ton $\mathrm{H}$ de la forme indéfinie, et qui constitue la seule trace de la présence du suffixe -ò dans la forme sous-jacente du mot.

Tableau 7. Suffixe $-\grave{o}$ avec les dissyllabes à syllabe finale Caa, Cee ou Coo

\begin{tabular}{|l|l|l|}
\hline Ind. & Def. & Exemples \\
\hline H H & H B & $\begin{array}{l}\text { búwáa / búwàa 'sorcier' } \\
\text { kóoráa / kóoràa 'kora' } \\
\text { sánkée / sáykèe 'moustiquaire' }\end{array}$ \\
\hline B H & B $\widehat{\mathrm{HB}}$ & $\begin{array}{l}\text { kòtóo / kòtôo 'aîné' } \\
\text { sàatée / sàatêe 'village' } \\
\text { tàmbáa / tàmbâa 'arbre, sp.' }\end{array}$ \\
\hline
\end{tabular}

7.1.4.2. Dissyllabes à syllabe finale Cii ou Cuu

Les deux premières syllabes de la forme définie présentent les mêmes tons que la forme indéfinie, et la troisième syllabe présente un ton $\mathrm{B}$ qui est le reflet direct du ton $\mathrm{B}$ de $-\grave{o}$. 
Tableau 8. Suffixe $-\grave{o}$ avec les dissyllabes à syllabe finale Cii ou Cuu

\begin{tabular}{|l|c|l|}
\hline Ind. & Def. & Exemples \\
\hline H H & H H B & $\begin{array}{l}\text { súlúu / súlúwòo 'hyène' } \\
\text { kítíi / kíitíyòo 'procès' } \\
\text { díntíi / díntíyòo 'qui a des enfants' }\end{array}$ \\
\hline B H & B H B & $\begin{array}{l}\text { kùrúu / kùrúwòo 'cola' } \\
\text { dòokúu / dòokúwòo 'travail' } \\
\text { kùntíi / kùntíyòo 'chef' }\end{array}$ \\
\hline
\end{tabular}

\subsubsection{Dissyllabes à syllabe finale CVN}

L'adjonction de -ò ne provoque aucun autre réajustement que la re-syllabification de la nasale finale du lexème.

Tableau 9. Suffixe $-\grave{o}$ avec les dissyllabes à syllabe finale CVN

\begin{tabular}{|l|c|l|}
\hline Ind. & Def. & Exemples \\
\hline H H & H H B & $\begin{array}{l}\text { kúlúy / kúlúyò 'bateau' } \\
\text { béeyáy / béeyáyò 'animal' } \\
\text { káykáy / káykáyò 'jardin' ou 'poussière' }\end{array}$ \\
\hline B H & B H B & $\begin{array}{l}\text { kùlúy / kùlúyò 'bateau' } \\
\text { làaráy / làaráyò 'lit'16 } \\
\text { kàykáy / kàykáyò 'écume' }\end{array}$ \\
\hline
\end{tabular}

\subsubsection{Lexèmes nominaux de trois syllabes ou plus}

Pour les lexèmes nominaux de trois syllabes ou plus, il y a la même relation entre ton final et ton pénultième que dans les dissyllabes. Pour les syllabes non-finales, il n'y a pas de restrictions autres que celles opérant de manière générale dans les limites du mot. Toutefois, statistiquement, on note une relative rareté des lexèmes dont le contour tonal de base présente une séquence $\mathrm{H} \mathrm{B}$ ailleurs qu'entre la syllabe pénultième et la finale.

${ }^{16}$ Le ton de ce terme est étymologiquement irrégulier, compte tenu de sa relation probable avec le verbe láa 'se coucher', mais cette irrégularité semble générale en mandinka. Certains locuteurs auxquels je faisais part de mon étonnement m'ont même proposé spontanément une paire minimale láaráy 'vêtement qu'on met pour la nuit' vs. làaráy 'lit'. 
7.1.5.1. Lexèmes nominaux de trois syllabes ou plus à syllabe finale légère

Tableau 10. Suffixe $-\grave{o}$ avec de noms à trois syllabes ou plus à syllabe finale légère

\begin{tabular}{|c|c|c|}
\hline Ind. & Def. & Exemples \\
\hline$\ldots \mathrm{HB}$ & $\ldots \mathrm{H} \mathrm{B}$ & $\begin{array}{l}\text { súgkútù / súgkútòo 'fille' } \\
\text { tùbáabù / tùbáabòo 'européen' } \\
\text { kùñámùcámù / kùñámùmcámòo 'folie' } \\
\text { kùllîimáarì / kùlîimáaròo 'arc-en-ciel' }\end{array}$ \\
\hline$\ldots \mathrm{B} \mathrm{H}$ & $\ldots \mathrm{B} \mathrm{HB}$ & $\begin{array}{l}\text { kàmbàaní / kàmbàanôo 'garçon' } \\
\text { kúgkúwùlú / kúykúwùlôo 'chacal' } \\
\text { kòotódùwá / kòotódùwôo 'oiseau, sp.' }\end{array}$ \\
\hline
\end{tabular}

7.1.5.2. Lexèmes nominaux de trois syllabes ou plus à syllabe finale lourde

Tableau 11. Suffixe $-\grave{o}$ avec de noms à de trois syllabes ou plus à syllabe finale Caa, $\mathrm{Cee}$ ou $\mathrm{Coo}$

\begin{tabular}{|c|c|l|}
\hline Ind. & Def. & Exemples \\
\hline$\ldots \mathrm{H} \mathrm{H}$ & $\ldots \mathrm{H} \mathrm{B}$ & $\begin{array}{l}\text { báráadáa / báráadàa 'théière' } \\
\text { nàsáaráa / nàsáaràa 'européen' }\end{array}$ \\
\hline$\ldots \mathrm{B} \mathrm{H}$ & $\ldots \mathrm{B} \mathrm{HB}$ & $\begin{array}{l}\text { sùrùwáa / sùrùwâa 'wolof' } \\
\text { bàtùutáa / bàtùutâa 'magicien' } \\
\text { bàtànsée / bàtànsêe 'aubergine' } \\
\text { bárìmbáa / bárìmbâa 'épouse de l'oncle maternel' } \\
\text { nìnkínàarée / nìnkínàarêe 'mousse' } \\
\text { săakùláa / săakùlâa 'sorte de héron' }\end{array}$ \\
\hline
\end{tabular}

Tableau 12. Suffixe $-\grave{o}$ avec de noms à de trois syllabes ou plus à syllabe finale Cii ou Cuu

\begin{tabular}{|c|c|l|}
\hline Ind. & Def. & Exemples \\
\hline$\ldots$ H H & $\ldots$ H H B & kòlòmbílíi / kòlòmbílíyòo 'margelle' \\
\hline$\ldots$ B H & $\ldots$ B H B & jàmbálùulúu / jàmbálùulúwòo 'plante, sp.' \\
\hline
\end{tabular}

Tableau 13. Suffixe $-\grave{o}$ avec de noms à de trois syllabes ou plus à syllabe finale CVN

\begin{tabular}{|c|c|l|}
\hline Ind. & Def. & Exemples \\
\hline ... H H & $\ldots$ H H B & $\begin{array}{l}\text { kámíyóy / kámíyóyò 'camion' } \\
\text { sàriyáy / sàríyáyò 'chariya' } \\
\text { kùnláaráy / kùnláaráyò 'coussin' }\end{array}$ \\
\hline ... B H & $\ldots$ B H B & $\begin{array}{l}\text { kàmiñáy / kàmiñáyò 'arbre, sp.' } \\
\text { káykàráy / káykàráyò 'toit' } \\
\text { săamèntéy / săamèntéyò 'lézard, sp.' } \\
\text { jàmbákàtáy / jàmbákàtáyò 'plante, sp.' }\end{array}$ \\
\hline
\end{tabular}




\subsection{Processus tonals dans la combinaison des noms avec leurs modifieurs}

\subsubsection{Remarques introductives}

Aucun processus tonal particulier ne s'observe dans les constructions « modifieur + nom », ainsi que dans les constructions «nom + modifieur » dans lesquelles le nom est à la forme définie. Par contre, dans les constructions « nom + modifieur » où le nom est à la forme du lexème nu (nom indéfini), il existe selon la nature du modifieur divers processus tonals qui ont comme résultat que la tonalité du nom modifié diffère de celle qui se manifeste lorsque le nom nu constitue à lui seul une expression nominale, sans que cela puisse s'expliquer par des règles phonologiques générales. Trois cas sont à distinguer.

\subsubsection{Constructions nom + modifieur à compacité tonale}

Les modifieurs du nom qui forment avec le nom une construction à compacité tonale sont essentiellement les adjectifs non dérivés, les adjectifs pourvus du suffixe sélectif -máa, et les adjectifs ordinaux dérivés de numéraux cardinaux au moyen du suffixe -ñjáy. On peut considérer que c'est aussi (au moins dans une certaine mesure) le cas du numéral kílìy, dont le cas sera traité à part.

7.2.2.1. Adjectifs non dérivés, adjectifs pourvus du suffixe sélectif -máa et adjectifs ordinaux

L'application de la règle de compacité tonale lorsqu'un nom est modifié par un adjectif non dérivé peut être illustrée par silà-wúléy 'singe rouge' < silá 'singe + wùléy 'rouge'.

L'application de la règle de compacité tonale lorsqu'un nom est modifié par un adjectif à la forme sélective peut être illustrée par jíi-kándí-màa 'l'eau qui est chaude' $<j i i$ 'eau' + kàndí 'chaud' + máa (sélectif) + ò (défini). ${ }^{17}$ Comme cela a déjà été noté pour le mandinka de Sédhiou, cette propriété tonale distingue le suffixe sélectif -máa du suffixe propriétif -máa (désigné comme « ornatif» dans (Creissels \& Sambou 2013)), cf. par exemple jíi kòomáa 'eau salée'.

Enfin, l'application de la règle de compacité tonale lorsqu'un nom est modifié par un adjectif ordinal peut être illustrée par kitàabù-fúláñjáy 'deuxième livre' < kitáabù 'livre' + fülànján 'deuxième'

\subsubsection{Le cas particulier de kílí 'un'}

Le numéral 'un' a la forme kílín lorsqu'il ne modifie pas un nom (notamment lorsqu'il est cité en isolation). Sa combinaison avec un nom qu'il modifie a en partie

${ }^{17}$ Il y a une différence sémantique entre jíikándòo 'de l'eau chaude' (jíi + kàndí $+-o$ o) et jíikándímàa (jíi + kàndí + máa + -ò) 'l'eau la plus chaude' ou 'la seule eau qui est chaude, parmi plusieurs eaux présentes dans la situation', mais tonalement, la règle de compacité s'applique dans les deux cas. 
les caractéristiques d'une construction à compacité tonale, mais en partie seulement. En effet, le nom modifié par kílín se comporte comme le premier constituant d'une construction à compacité tonale, avec une tonalité entièrement basse si son ton initial est $\mathrm{B}$, et une tonalité entièrement haute si son ton initial est $\mathrm{H}$. Par contre, kílíy en tant que modifieur de nom prend la tonalité kílìn, alors que dans une construction à compacité tonale régulière, sa tonalité ne devrait pas être modifiée. On aura ainsi par exemple :

tùbáabù 'européen' + kílín 'un' $\rightarrow$ tùbàabù kílìn

káykàráy 'toit' + kílín 'un' $\rightarrow$ káykáráy kílì

\subsubsection{Constructions nom + modifieur mettant en jeu une règle de pont tonal}

La règle de pont tonal, qu'on peut schématiser comme $\mathrm{B} \rightarrow \mathrm{H} / \mathrm{H}_{-} \# \mathrm{H}$, affecte la syllabe finale des noms à la forme nue suivis de certains modifieurs. Compte tenu des schèmes tonals possibles pour les lexèmes nominaux, cette règle concerne les noms dont la syllabe finale est une syllabe légère à ton bas.

Cette règle est notamment déclenchée par l'indéfini dóo :

$\begin{array}{lll}\text { básà } & + \text { dóo } \rightarrow \text { básá dóo } & \text { (lézard) } \\ \text { băanà } & + \text { dóo } \rightarrow \text { băaná dóo } & \text { (arbre, sp.) } \\ \text { súnkútù } & + \text { dóo } \rightarrow \text { súnkútú dóo } & \text { (fille) } \\ \text { tùbáabù } & + \text { dóo } \rightarrow \text { tùbáabú dóo } & \text { (Européen) } \\ \text { kùlîimáarì } & + \text { dóo } \rightarrow \text { kùlîimáarí dóo } & \text { (arc-en-ciel) }\end{array}$

La même règle opère lorsqu'un nom à la forme nue est modifié par un adjectif qui ne forme pas une construction à compacité tonale avec sa tête (par exemple, kitáabù 'livre' + fûurín 'sans valeur' $\rightarrow$ kitáabú fûurín), ou lorsqu'un nom à la forme nue est modifié par dántày 'quelques-uns' (par exemple, kitáabù 'livre' + dántày $\rightarrow$ kitáabú dántày).

7.2.4. Constructions nom + modifieur mettant en jeu une règle d'insertion d'un ton bas

En mandinka du Pakaawu, les numéraux à ton initial $\mathrm{H}$ autres que 'un' (náani 'quatre', lúulù 'cinq', wóorò 'six', etc.) forment avec le nom qu'ils modifient une construction dont la particularité est de provoquer une modification tonale du nom modifié qui peut se décrire comme l'insertion d'un ton $\mathrm{B}$. Cette règle concerne les noms monosyllabiques CVVN, les noms monosyllabiques CVV ou CVN à ton de base $\mathrm{H}$, et les noms de deux syllabes ou plus dont la syllabe finale est une syllabe lourde à ton $\mathrm{H}$. Dans tous les cas, le ton B s'associe à la more finale du nom, créant un ton modulé $\widehat{\mathrm{HB}}$ (et dans le cas des monosyllabes lourds, un ton doublement modulé $\widehat{\mathrm{BHB}}$ ) : 


\begin{tabular}{|c|c|c|c|}
\hline tǔuy & + náanì & $\rightarrow$ tùûy náanì & (termitière) \\
\hline báa & + náanì & $\rightarrow$ bâa náanì & (fleuve) \\
\hline súu & + náanì & $\rightarrow$ sûu náanì & (maison) \\
\hline bún & + náani & $\rightarrow$ bûy náanì & (chambre) \\
\hline ́́oráa & + náanì & $\rightarrow$ kóorâa náanì & (kora) \\
\hline atée & + náanì & $\rightarrow$ sàatêe náanì & (village) \\
\hline lún & + náanì & $\rightarrow$ kúlûy náanì & (bateau) \\
\hline lóy & + náanì & $\rightarrow$ kòlôy náanì & (puits) \\
\hline nláaráy & + náanì & $\rightarrow$ kùnláarây náanì & (coussin) \\
\hline ràmbún & + náanì & $\rightarrow$ kàràmbûy náanì & (école) \\
\hline
\end{tabular}

Les noms ayant une autre structure syllabo-tonale présentent par contre avec les numéraux autres que 'un' leur tonalité de base, avec simplement l'application éventuelle de la règle de relèvement tonal si le numéral commence par un ton $\mathrm{B}$, cf. par exemple tùbáabù náanì 'quatre Européens' / tùbáabú fùlá 'deux Européens'.

\subsubsection{Récapitulation}

Selon leur interaction tonale avec les modifieurs qui peuvent leur être postposés, les lexèmes nominaux se répartissent en trois groupes.

7.2.5.1. Lexèmes nominaux qui ne subissent aucune modification tonale dans les constructions 'nom + modifieur' autres que celles à compacité tonale

Font partie de cette catégorie les monosyllabes $\mathrm{CVV}$ et $\mathrm{CVN}$ à ton $\widehat{\mathrm{BH}}$ (comme sŭu 'cheval' ou sŭy 'voleur'), ainsi que les noms de deux syllabes ou plus qui ont une syllabe finale légère et un ton B sur la syllabe pénultième (comme mùsú 'femme', cf. par exemple mùsú dóo 'une femme', mùsú náanì 'quatre femmes').

7.2.5.2. Lexèmes nominaux soumis à la règle d'insertion d'un ton $B$

Font partie de cette catégorie les monosyllabes supra-lourds, les monosyllabes à ton $\mathrm{H}$, et les noms de deux syllabes ou plus à syllabe finale lourde (comme sàatée 'village', cf. sàatée dóo 'un village', sàatêe náanì 'quatre villages'.

7.2.5.3. Lexèmes nominaux soumis à la règle de pont tonal

Font partie de cette catégorie les noms de deux syllabes ou plus à syllabe finale légère et à ton $\mathrm{H}$ ou $\widehat{\mathrm{BH}}$ sur la pénultième (comme básà 'lézard, cf. básá dóo 'un lézard', básà náani 'quatre lézards'.

\section{Le ton dans le système verbal}

\subsection{Classes tonales de lexèmes verbaux}

\subsubsection{Remarques introductives}

De manière analogue à la décision prise pour les lexèmes nominaux, on considère dans cet article que le contour tonal de base des lexèmes verbaux est celui que 
manifeste le lexème verbal dépourvu de tout suffixe lorsqu'il occupe la position $\mathrm{V}$ dans le schème de prédication $\mathrm{S} p(\mathrm{O}) \mathrm{V} \mathrm{X}$, dans un contexte phonologique où le relèvement tonal n'opère pas (c'est-à-dire, si le verbe à la forme nue précède immédiatement une pause ou un mot à ton initial $\mathrm{H}$ ). Ce ton est d'ailleurs identique à celui observé lorsque les lexèmes verbaux sont cités en isolation à l'infinitif (ce qui est pour les locuteurs du mandinka la forme de citation spontanée des lexèmes verbaux).

Cette décision, tout en étant à la fois naturelle et totalement cohérente avec celle prise pour les lexèmes nominaux, oblige à admettre un contraste important entre les schèmes tonals possibles pour les lexèmes nominaux et ceux possibles pour les lexèmes verbaux. Il s'agit d'une situation relativement courante dans les langues d'Afrique subsaharienne, mais totalement inattendue dans un parler mandingue, et d'ailleurs, lors de l'enquête qui a abouti à cet article, j'ai mis longtemps à accepter la nécessité de cette analyse.

En mandinka du Pakaawu, dans les contextes considérés comme pertinents pour l'identification du ton de base, les verbes à la forme nue de deux syllabes ou plus dont la syllabe finale est lourde peuvent seulement avoir un ton $\widehat{\mathrm{HB}}$ sur leur syllabe finale, alors que dans des contextes comparables, les noms à la forme nue de deux syllabes ou plus dont la syllabe finale est lourde peuvent seulement avoir un ton $\mathrm{H}$ sur leur syllabe finale.

Après avoir passé en revue les schèmes tonals possible pour les lexèmes verbaux de deux syllabes ou plus, nous examinerons ce qui se passe avec les lexèmes verbaux monosyllabiques, dont le comportement tonal présente quelques problèmes.

\subsubsection{Lexèmes verbaux de deux syllabes ou plus}

Au-delà de deux syllabes, en plus des contraintes générales sur les séquences tonales dans les limites du mot, on observe pour les lexèmes verbaux deux contraintes supplémentaires :

- aucun lexème verbal ne présente de séquence $\mathrm{H}$ B en position interne ;

- à une exception près (bǔutèe 'frapper'), seul le ton $\widehat{\mathrm{HB}}$ est attesté sur la syllabe finale des verbes à syllabe finale lourde.

8.1.2.1. Lexèmes verbaux dissyllabiques à syllabe finale légère

Il y a deux contours tonals de base possibles ( $\mathrm{H} \mathrm{B}$ et $\mathrm{B} \mathrm{H}$ ) pour les lexèmes verbaux dissyllabiques constitués de deux syllabes légères, et un troisième $(\widehat{\mathrm{BH}} \mathrm{B})$ pour ceux dont la première syllabe est lourde et la deuxième légère.

- Le schème H B peut être illustré par kili 'appeler', kárà 'coudre’, báayì 'annuler', háañi 'oser', fúntì 'sortir', dánkà 'maudire'.

- Le schème B H peut être illustré par mùtá 'saisir', bòri 'courir', nàatí 'apporter', jàará 'soigner', dàmfú 'donner un coup de pied', kùntú 'couper'. 
- Le schème $\widehat{\mathrm{BH}} \mathrm{B}$ peut être illustré par jăabì 'répondre', tǔubi 'convertir'.

8.1.2.2. Lexèmes verbaux dissyllabiques à syllabe finale lourde

Il y a deux contours tonals de base possibles $(\widehat{H ~} \widehat{\mathrm{HB}}$ et $\mathrm{B} \widehat{\mathrm{HB}})$ pour les lexèmes verbaux dissyllabiques constitués d'une première syllabe légère et d'une deuxième syllabe lourde ; un troisième schème ( $\widehat{\mathrm{BH}} \mathrm{B})$ est attesté, mais avec un verbe seulement.

- Le schème H $\widehat{\mathrm{HB}}$ peut être illustré par fútûu 'épouser', firîy 'détacher', súutêe 'reconnaître', jíibôy 'arroser', bindêe 'sarcler', kénsûy 'rassembler'.

- Le schème B $\widehat{\mathrm{HB}}$ peut être illustré par bàtâa 'se fatiguer', jòlôy 'tomber', dèemâa 'aider', kùurây 'tomber malade', kùmbôo 'pleurer, kòntôy 'saluer'.

- Le schème $\widehat{\mathrm{BH}} \mathrm{B}$ est attesté seulement par bǔutèe 'frapper'.

8.1.2.3. Lexèmes verbaux trisyllabiques à syllabe finale légère

$\mathrm{Il} \mathrm{y}$ a trois schèmes tonals possibles $(\mathrm{H} \mathrm{H} \mathrm{B,} \mathrm{B} \mathrm{H} \mathrm{B} \mathrm{et} \mathrm{B} \mathrm{B} \mathrm{H)} \mathrm{pour} \mathrm{les} \mathrm{lexèmes}$ verbaux trisyllabiques à syllabe finale légère :

- Le schème H H B peut être illustré par dárájà 'être populaire', fitínà 'se quereller'.

- Le schème B HB peut être illustré par dàbárì 'ensorceler', fàháamù 'comprendre'.

- Le schème B B H peut être illustré par dìyàamú 'parler', fàabàndí 'tenir compagnie'.

8.1.2.4. Lexèmes verbaux trisyllabiques à syllabe finale lourde

Il y a trois schèmes tonals possibles $(\mathrm{H} \mathrm{H} \mathrm{HB}, \mathrm{B} H \widehat{\mathrm{HB}}$ et $\mathrm{B} B \mathrm{HB})$ pour les lexèmes verbaux trisyllabiques à syllabe finale lourde :

- Le schème H H HB peut être illustré par báláfâa 'avoir pitié', dándálâa 'mettre en garde'.

- Le schème B H HB peut être illustré par màanéenêe 'amadouer'.

- Le schème B B Н̂ peut être illustré par ñinìnkâa 'interroger', sitìkôy 'envoûter'.

\subsubsection{Lexèmes verbaux monosyllabiques}

8.1.3.1. Lexèmes verbaux monoyllabiques : le cas général

En règle générale, les lexèmes verbaux monosyllabiques présentent invariablement une forme $\mathrm{CVV}$ ou CVN, avec deux schèmes tonals de base possibles, $\widehat{\mathrm{HB}}$ et $\widehat{\mathrm{BH}}$ :

- Le schème $\widehat{\mathrm{HB}}$ peut être illustré par dîi 'donner', fêe 'souffler', bûy 'viser', kôy 'détester', etc.

- Le schème $\widehat{\mathrm{BH}}$ peut être illustré par făa 'mourir', tăa 'prendre', bǒy 'verser', săy 'acheter', etc. 
8.1.3.2. Cas particulier 1: lexèmes verbaux monosyllabiques qui ont un allomorphe $\mathrm{CV}$

Il s'agit de six lexèmes verbaux ( $k e ́$ ' faire', je’ 'voir', só 'donner', bó 'sortir', fó 'dire' et tu' 'laisser') qui ont un allomorphe CV lorsqu'ils fonctionnent en tant que verbes, soit à la forme nue, soit combinés aux suffixes -tá et -lá', et un allomorphe CVV (à ton $\widehat{\mathrm{HB}}$ ) dans tous les autres cas.

Comme cela a déjà été discuté en 6.6.1, en l'absence de suffixe, l'allomorphe CV présente une alternance tonale qu'on peut expliquer par un schème tonal sous-jacent Hb. L'exemple (31) illustre cette alternance, qui produit un effet de polarité tonale lorsque le mot suivant est à ton initial $\mathrm{H}$.
a. $\dot{A}$
só
kódòo lá!
3SG donner argent.D POSTP
'Donne-lui l'argent !'
b. म́ sò kódòo lá!
1SG donner argent.D POSTP
'Donne-moi l'argent !'

8.1.3.3. Cas particulier 2 : táa 'aller' et autres verbes monosyllabiques en aa

Dans toute l'enquête dont cet article livre les résultats, le comportement tonal des verbes monosyllabiques en $a a$ pour lesquels on attendrait un ton $\widehat{\mathrm{HB}}$ est à peu près le seul point sur lequel j'ai noté des flottements dans les productions spontanées du consultant, ainsi que des hésitations dans ses jugements d'acceptabilité.

Le seul cas parfaitement net est celui de táa 'aller'. Contrairement aux régularités générales, chez le consultant, ce verbe présente de manière invariable des réalisations tonales $\mathrm{H}$, jamais $\widehat{\mathrm{HB}}$. Avec les autres verbes en $a$ a pour lesquels un ton $\widehat{\mathrm{HB}}$ est attendu (par exemple lâa/láa 'se coucher' ou fâa/fáa 's'emplir'), on observe des flottements.

\subsection{Propriétés tonales du suffixe d'accompli positif intransitif -tá}

Ce suffixe présente des variations tonales semblables à celles décrites en 6.6 pour les verbes CV ainsi que pour des enclitiques qu'il a été proposé d'analyser comme ayant une structure tonale sous-jacente $\mathrm{Hb}$. En effet, s'il succède à un lexème verbal se terminant par un ton $\mathrm{B}$ ou $\widehat{\mathrm{HB}}$, il est invariablement $\mathrm{H}$, alors que s'il succède à un lexème verbal se terminant un ton $\mathrm{H}$ ou $\widehat{\mathrm{BH}}$, il présente un ton bas, qui peut toutefois laisser la place à un $\mathrm{H}$ selon la règle de relèvement tonal.

\begin{tabular}{|c|c|c|c|}
\hline$d \hat{i} i$ & $+-t a ́$ & $\rightarrow d \hat{i} \mathbf{i}-t a ́$ & (donner) \\
\hline$\tilde{n} \hat{u} \eta$ & $+-t a ́ n$ & $\rightarrow \tilde{n} \hat{u} n-t a ́$ & (se charger) \\
\hline bmò & $+-t a ́ n$ & $\rightarrow$ dómò-tá & (manger) \\
\hline jăabì & $+-t a ́ n$ & $\rightarrow$ jăabì-tá & (répondre) \\
\hline
\end{tabular}




\begin{tabular}{|c|c|c|c|c|}
\hline bàtâa & $+-t a ́$ & $\rightarrow$ bàtâa-tá & (se fatiguer) & \\
\hline jòlôy & $+-t a ́$ & $\rightarrow$ jòlôn-tá & (tomber) & \\
\hline fitínà & $+-t a ́ n$ & $\rightarrow$ fitinà-tá & (se quereller) & \\
\hline dàbárì & $+-t a ́$ & $\rightarrow$ dàbárì-tá & (ensorceler) & \\
\hline ñinìnkâa & $+-t a ́$ & $\rightarrow$ ñinìnkâa-tá & (interroger) & \\
\hline$k e ́$ & $+-t a ́$ & $\rightarrow k e ́-t a ̀(\sim k e ́-t c$ & $a ́$ devant B) & (faire) \\
\hline năa & $+-t a ́ n$ & 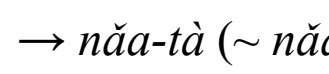 & a-tá devant B) & (venir) \\
\hline fùtá & $+-t a ́$ & $\rightarrow$ fùtá-tà $(\sim$ fùt & tá-tá devant B) & (arriver) \\
\hline lìyàamú & $+-t a ́$ & $\rightarrow$ dìyàamú-tà ( & ( dìyàamú-tá devant $\mathrm{B})$ & (parler) \\
\hline
\end{tabular}

En outre, avec les lexèmes verbaux de deux syllabes ou plus dont la tonalité structurelle se termine par une séquence $\mathrm{H} \widehat{\mathrm{HB}}$, au contact du suffixe -tá (réalisé -tá), le ton $\widehat{\mathrm{HB}}$ à la finale du lexème laisse la place à un ton $\mathrm{B}$. Ceci peut s'expliquer comme un réajustement motivé par la contrainte selon laquelle une syllabe à ton $\widehat{\mathrm{HB}}$ peut succéder immédiatement à un ton $\mathrm{H}$ en fin de mot, mais pas en position non finale.

\begin{tabular}{|c|c|c|c|}
\hline tíñâa & $+-t a ́ n$ & $\rightarrow$ tíñàa-tá & (abîmer) \\
\hline áñjây & $+-t a a^{\prime}$ & $\rightarrow$ jáñjàn-tá & (se disperser) \\
\hline & $+-t a^{\prime}$ & $\rightarrow$ báláfàa-tá & (avoir pitié) \\
\hline iây & $+-t a ́$ & $\rightarrow$ náaniñjàn-tá & (se produire une 4ème fois) \\
\hline
\end{tabular}

\subsection{Propriétés tonales du suffixe d'infinitif $-l^{\prime}{ }^{\prime}$}

Le suffixe d'infinitif-lá a exactement les mêmes propriétés tonales que -tá ${ }^{\prime}$ : tous les exemples énumérés à la section 8.2 conservent exactement leur tonalité si on remplace -tá par-lá .

\subsection{Propriétés tonales du suffixe résultatif -ríy}

Le suffixe -ríy a un ton invariablement $\mathrm{H}$, et de ce point de vue il ressemble aux suffixes dérivatifs qui, comme c'est généralement en mandingue, forment avec leur base une construction à compacité tonale. Toutefois, en mandinka du Pakaawu, le suffixe résultatif interagit de façon spécifique avec sa base. En outre, contrairement à ce qui est suggéré dans Creissels \& Sambou (2013) pour le parler de Sédhiou, le fait que la forme résultative soit employée verbalement ou adjectivalement n'a aucune incidence sur sa tonalité.

$\mathrm{Au}$ contact du suffixe résultatif, la tonalité des monosyllabes $\widehat{\mathrm{HB}}$ ainsi que des bases verbales dont le schème tonal structurel se termine par une séquence $\mathrm{H} \mathrm{B}$ ou $\mathrm{B}$ $\widehat{\mathrm{HB}}$ ne subit aucune modification.

$$
\begin{aligned}
& \text { sî }+ \text {-rín } \rightarrow \text { sî̀-rí } \quad \text { (atteindre) } \\
& \text { tábì } \quad+\text {-rín } \rightarrow \text { tábì-rín (cuire) } \\
& \text { jăabi }+ \text {-rín } \rightarrow \text { jăabì-rín (répondre) }
\end{aligned}
$$




$$
\begin{aligned}
& \text { jòlôn }+ \text {-rín } \rightarrow \text { jòlôn-dín (tomber) } \\
& \text { dàbárì + -rín } \rightarrow \text { dàbárì-lín (ensorceler) } \\
& \text { dárájà }+ \text {-rín } \rightarrow \text { dárájà-rín (être populaire) }
\end{aligned}
$$

$\mathrm{Au}$ contact du suffixe résultatif, les monoyllabes $\widehat{\mathrm{BH}}$ prennent un ton $\mathrm{B}$, et le ton $\mathrm{H}$ final des bases verbales de deux syllabes ou plus se terminant par une séquence tonale B H est remplacé par un ton B.

$$
\begin{aligned}
& \text { sii } \quad+\text {-rí } \rightarrow \text { sì-rín (s'asseoir) } \\
& \text { jàni } \quad+\text {-rín } \rightarrow \text { jànì-rín (brûler) } \\
& \text { bàràmá + -rín } \rightarrow \text { bàràmà-rín (blesser) }
\end{aligned}
$$

Enfin, avec les lexèmes verbaux de deux syllabes ou plus dont la tonalité structurelle se termine par une séquence $\mathrm{H} \widehat{\mathrm{HB}}$, exactement comme au contact $\mathrm{du}$ suffixe -tá , le ton $\widehat{\mathrm{HB}}$ à la finale du lexème laisse la place à un ton $\mathrm{B}$, ce qui peut s'expliquer comme motivé par la contrainte selon laquelle une syllabe à ton $\widehat{\mathrm{HB}}$ en position interne de mot ne peut pas succéder immédiatement à un ton $\mathrm{H}$.

$$
\begin{aligned}
& \text { tínâa + -rín } \rightarrow \text { tínàa-rín (abîmer) } \\
& \text { jáñjây + -rín } \rightarrow \text { jáñjàn-dín (se disperser) }
\end{aligned}
$$

\section{Conclusion}

Dans cet article, $\mathrm{j}$ 'ai proposé une description du système tonal du mandinka du Pakaawu en insistant sur la façon dont ce système articule des phénomènes tonals couramment rencontrés en mandingue et d'autres spécifiques de ce parler. J'ai notamment souligné l'importance du poids syllabique dans le conditionnement des phénomènes tonals, et analysé en détail un système d'alternances tonales systématiques à la finale des mots très différent de ce qui a déjà été décrit en mandingue. J'ai notamment conclu à la nécessité de poser que les contours tonals de base possibles pour les lexèmes verbaux à syllabe finale lourde sont différents de ceux possibles pour les lexèmes nominaux de structure syllabique identique.

La question que je voudrais évoquer en guise de conclusion est la signification qu'il convient d'accorder aux divergences entre le système décrit dans cet article et la description du système tonal du mandinka de Sédhiou proposée dans Creissels \& Sambou (2013).

Le système tonal du mandinka de Sédhiou diffère indiscutablement de celui du mandinka du Pakaawu sur au moins un point important. Il existe en effet à Sédhiou un phénomène de downstep se produisant dans des conditions plutôt mystérieuses, au sens où rien de ce qui a pu être observé ailleurs en mandingue ne peut être mis en relation avec l'apparition de ce downstep (Creissels \& Sambou 2013: 48-49, 179-186). Or rien de semblable ne s'observe en mandinka du Pakaawu. Par exemple, on voit en (32) que 
dans un contexte où rien ne suggère de postuler la présence d'un ton $B$ flottant, en mandinka de Sédhiou, le ton $\mathrm{H}$ de la deuxième syllabe de Fàatú est prononcé nettement plus haut que le ton $\mathrm{H}$ initial du mot suivant, alors qu'en mandinka du Pakaawu, conformément à la règle générale pour les tons $\mathrm{H}$ en position faible, il est abaissé à un niveau moyen.
a. Fàatú 'kúmándí này!
Fatou appeler VEN
'Demande à Fatou de venir ici !' (Sédhiou)

b. Fàatú kúmándí năy! phonétiquement : [fà:tū kúmándí này]

Fatou appeler VEN

'Demande à Fatou de venir ici !' (Pakaawu)

Par contre, il convient de relativiser les autres divergences entre le système décrit ici et celui proposé pour le mandinka de Sédhiou dans Creissels \& Sambou (2013). Dans l'enquête sur le parler de Sédhiou, nous avons été confrontés à un certain nombre de variations au niveau tonal. Ces variations n'ont rien de surprenant compte tenu du fait que Sédhiou est une grande ville, caractérisée depuis sa fondation au milieu du 19ème siècle par un brassage constant de populations originaires de toutes les régions de Casamance et de Guinée Bissau. Le problème est que nous avons probablement eu le tort de vouloir ramener ces variations à un système unique, et qu'en outre nous n'avons pas envisagé un éventail suffisamment large d'hypothèses susceptibles de les expliquer. Nous avons abandonné probablement un peu trop vite l'idée (un moment envisagée) que des lexèmes dont le contour tonal de base ailleurs en mandingue est ... H H puissent avoir un contour tonal de base ... H B, et nous n'avons même pas envisagé par exemple la possibilité que les syllabes lourdes à la finale des lexèmes verbaux puissent avoir des propriétés tonales différentes de celles à la finale des lexèmes nominaux, ou encore que certaines alternances puissent mettre en jeu un phénomène de pont tonal. Nous avons essayé tant bien que mal de rendre compte de l'ensemble des variations tonales en fin de mot en posant une règle générale convertissant les séquences HH en HB devant pause et une variation libre entre $\widehat{\mathrm{HB}}$ et $\mathrm{B}$ pour les syllabes lourdes en fin de mot après ton $\mathrm{H}$. Toutefois, en réécoutant maintenant les enregistrements réalisés à Sédhiou, il m'apparaît qu'il a fallu pour cela traiter implicitement comme marginales ou accidentelles un certain nombre de données qui par contre entrent parfaitement dans le cadre de ce qui est décrit ici pour le mandinka du Pakaawu. En fait, en ce qui concerne les alternances tonales en fin de mot et leur analyse, ma conviction est maintenant que la description tonale $d u$ mandinka de Sédhiou devrait être revue en partant de l'hypothèse qu'une partie au moins des locuteurs puisse avoir un système tonal beaucoup plus proche de celui décrit ici que de celui que nous avons proposé dans la grammaire de 2013. 


\section{Abréviations}

Les abréviations suivantes sont utilisées dans la description des processus tonals :

$\mathrm{H}$ : ton haut

$\mathrm{h}:$ ton haut flottant

B : ton bas

$\mathrm{b}:$ ton bas flottant

$\widehat{\mathrm{HB}}$ : ton complexe haut-bas

$\widehat{\mathrm{BH}}$ : ton complexe bas-haut

$\widehat{\mathrm{BHB}}$ : ton complexe bas-haut-bas

$\mathrm{H}^{\mathrm{x}}$ : un ou plusieurs tons hauts successifs

$\mathrm{B}^{\mathrm{x}}$ : un ou plusieurs tons hauts successifs

!H : ton haut abaissé

\#\# : pause

\# : limite entre deux mots non marquée par une pause

$=:$ limite entre un clitique et son hôte

Les abréviations suivantes sont utilisées pour la schématisation des structures syllabiques

$\mathrm{C}:$ consonne quelconque

$\mathrm{V}$ : voyelle quelconque

$\mathrm{VV}$ : voyelle longue quelconque

$\mathrm{N}$ : consonne nasale en position de coda (analysable comme non spécifiée quant à son lieu d'articulation, car celui-ci dépend entièrement de ce qui suit ; devant pause, sa réalisation par défaut est celle d'une nasale vélaire $\eta$ )

Enfin, les abréviations suivantes sont utilisées pour le glosage des exemples :

ABSTR $=$ suffixe dérivatif d'abstraction

$\mathrm{ACP}=$ accompli

COPLOC $=$ copule de localisation

$\mathrm{D}=$ défini

$\mathrm{FOC}=$ focalisation

GEN = génitif

INACP $=$ inaccompli

INDEF $=$ indéfini

INTR $=$ intransitif

LOC $=$ locatif

$\mathrm{NEG}=$ négation

$\mathrm{PL}=$ pluriel

POSTP $=$ postposition 
POT $=$ potentiel

$\mathrm{PROH}=$ prohibitif

QUOT $=$ quotatif

$\mathrm{SG}=$ singulier

$\mathrm{SBJF}=$ subjonctif

SPHP $=$ postposition 'dans la sphère personnelle de'

$\mathrm{TR}=$ transitif

$\mathrm{VEN}=$ vénitif

\section{Références bibliographiques}

Creissels, Denis. 2009. Le malinké de Kita (Mande languages and linguistics 9). Rüdiger Köppe Verlag. Köln.

Creissels, Denis. 2016. Phonologie segmentale et tonale du soninké (parler du Kingi). Mandenkan 55. 3-174.

Creissels, Denis \& Claire Grégoire. 1993. La notion de ton marqué dans l'analyse d'une opposition tonale binaire: Le cas du mandingue. Journal of African Languages and Linguistics 14(2). 107-154.

Creissels, Denis \& Pierre Sambou. 2013. Le mandinka. Phonologie, grammaire, textes. Paris: Karthala.

Dramé, Yaya. 2016. Propriétés phonologiques et morphosyntaxiques des noms propres de personne en mandinka du Pakaawu. Lyon: Université Lumière Lyon2 Mémoire de master.

Dumestre, Gérard. 2003. Grammaire fondamentale du bambara. Paris: Karthala.

Green, Christopher R. 2015. The foot domain in Bambara. Language 91(1). e1e26.

Green, Christopher R., Jonathan C. Anderson \& Samuel G. Obeng. 2013. Interacting tonal processes in Susu. Mandenkan 50. 61-84. doi:10.4000/mandenkan.876.

Grégoire, Claire. 1986. Le maninka de Kankan. Eléments de description phonologique (Annales). Tervuren: Musée Royal de l'Afrique Centrale.

Roche, Christian. 1985. Histoire de la Casamance, conquête et résistance : 18501920. Paris: Karthala.

Rowlands, E.C. 1959. A grammar of Gambian Mandinka. London: SOAS: University of London.

Schaffer, Matthew. 2003. Djinns, stars and warriors: Mandinka legends from Pakao, Senegal (African Sources for African History 5). Leiden - Boston: Brill.

Vydrin, Valentin. 2017. Bamana jazyk (Бамана язык) [Bamana]. In Valentin Vydrin, Yulia Mazurova, Andrej Kibrik \& Elena Markus (eds.), Jazyki mira: Jazyki mande (Языки мира: Языки манде) [Languages of the world: Mande languages], 46-143. St. Petersburg: Nestor-Historia. 


\section{Tonologie du mandinka du Pakaawu}

Cet article décrit le système tonal du mandinka du Pakaawu. Ce parler se caractérise notamment par l'importance du poids syllabique dans le conditionnement des phénomènes tonals et par des alternances tonales systématiques à la finale des mots très différentes de ce qui a été décrit dans les autres parlers mandingues, conduisant notamment à poser que les mots se terminant par une syllabe légère ne peuvent pas avoir un contour tonal de base se terminant par deux syllabes successives à ton haut, et que les contours tonals de base possibles pour les lexèmes verbaux à syllabe finale lourde sont différents de ceux possibles pour les lexèmes nominaux de structure syllabique identique.

Mots-clés: mandé, mandingue, mandinka, alternances tonales, poids syllabique.

\section{Tonology of the Pakaawu Mandinka}

This article describes the tonal system of Pakaawu Mandinka. This Manding variety is characterized by the importance of syllable weight in the conditioning of tonal phenomena, and by systematic tonal alternations affecting the last syllable of words that differ in important respects from what has been described so far for other Manding varieties, leading to posit that words ending with a light syllable cannot have a basic tonal contour ending with two successive H-toned syllables, and that the possible basic tonal contours of verbal lexemes ending with a heavy syllable are different from those possible for the nominal lexemes having the same syllabic structure.

Keywords: Mande, Manding, Mandinka, tonal alternations, syllable weight.

\section{Тонология мандинка р-на Пакау}

В статье описывается тональная система мандинка района Пакау. Этот диалект характеризуется, в частности, значимостью слогового веса как фактора, обуславливающего тональные явления, а также систематическими тональными чередованиями в конце слов, радикально отличающимися от тех, которые наблюдаются в других идиомах манден. В частности, слова с конечным лёгким слогом не могут иметь базовый тональный контур, заканчивающийся последовательностью двух высокотоновых слогов. Базовые тональные контуры, допустимые для глагольных лексем с конечным тяжёлым слогом, отличаются от контуров, допустимых для именных лексем аналогичной слоговой структуры.

Ключевые слова: манде, манден, мандинка, тоновые чередования, слоговой вес 